\title{
Review
}

\section{The thalamocortical vestibular system in animals and humans}

\author{
Christophe Lopez ${ }^{a, b, *}$, Olaf Blanke $e^{a, c}$
}

${ }^{a}$ Laboratory of Cognitive Neuroscience, Brain-Mind Institute, Ecole Polytechnique Fédérale de Lausanne, Swiss Federal Institute of Technology, Lausanne, Switzerland

${ }^{\mathrm{b}}$ Institut für Psychologie, Abteilung für Kognitive Psychologie, Wahrnehmung und Methodenlehre, Universität Bern, Bern, Switzerland 'Department of Neurology, University Hospital, Geneva, Switzerland

\section{A R T I C L E I N F O}

\section{Article history:}

Accepted 30 December 2010

Available online 9 January 2011

\section{Keywords:}

Vestibular cortex

Multisensory integration

Neuroimagery

Electrophysiology

Neurology

Caloric and galvanic vestibular

stimulation

\begin{abstract}
A B S T R A C T
The vestibular system provides the brain with sensory signals about three-dimensional head rotations and translations. These signals are important for postural and oculomotor control, as well as for spatial and bodily perception and cognition, and they are subtended by pathways running from the vestibular nuclei to the thalamus, cerebellum and the "vestibular cortex." The present review summarizes current knowledge on the anatomy of the thalamocortical vestibular system and discusses data from electrophysiology and neuroanatomy in animals by comparing them with data from neuroimagery and neurology in humans. Multiple thalamic nuclei are involved in vestibular processing, including the ventroposterior complex, the ventroanterior-ventrolateral complex, the intralaminar nuclei and the posterior nuclear group (medial and lateral geniculate nuclei, pulvinar). These nuclei contain multisensory neurons that process and relay vestibular, proprioceptive and visual signals to the vestibular cortex. In non-human primates, the parieto-insular vestibular cortex (PIVC) has been proposed as the core vestibular region. Yet, vestibular responses have also been recorded in the somatosensory cortex (area 2v, 3av), intraparietal sulcus, posterior parietal cortex (area 7), area MST, frontal cortex, cingulum and hippocampus. We analyze the location of the corresponding regions in humans, and especially the human PIVC, by reviewing neuroimaging and clinical work. The widespread vestibular projections to the multimodal human PIVC, somatosensory cortex, area MST, intraparietal sulcus and hippocampus explain the large influence of vestibular signals on self-motion perception, spatial navigation, internal models of gravity, one's body perception and bodily self-consciousness.
\end{abstract}

() 2011 Elsevier B.V. All rights reserved.

\footnotetext{
Abbreviations: 3aHv, 3a-hand-vestibular area; 3aNv, 3a-neck-vestibular area; ASS, anterior suprasylvian cortex; DVN, descending vestibular nucleus; FEF, frontal eye fields; Ig, insula granularis; IL, intralaminar nuclei; LD, lateral dorsal nucleus; LGN, lateral geniculate nucleus; LP, lateral posterior nucleus; LVN, lateral vestibular nucleus; MGmc, medial geniculate nucleus, pars magnocellularis; MGN, medial geniculate nucleus; MIP, medial intraparietal area; MST, medial superior temporal area; MT, middle temporal area; MVN, medial vestibular nucleus; PIVC, parieto-insular vestibular cortex; PO, posterior group of the thalamus; Reipt, area retroinsularis pars parietalis; Ri, area retroinsularis; SGN, suprageniculate nucleus; SVN, superior vestibular nucleus; TPJ, temporo-parietal junction; VA, ventroanterior thalamic nucleus; Vim, nucleus ventralis intermedius; VIP, ventral intraparietal area; VL, ventrolateral thalamic nucleus; VP, ventro-posterior thalamus; VPI, ventral posterior inferior nucleus; VPL, ventral posterior lateral nucleus; VPM, ventral posterior medial nucleus; VPP, ventral posterior nucleus, pars posterior; VPS, visual posterior sylvian area

* Corresponding author. University of Berne, Institut für Psychologie, Abteilung für Kognitive Psychologie, Wahrnehmung und Methodenlehre, Muesmattstrasse, 45. 3012 Bern, Switzerland.

E-mail addresses: christophe.lopez@psy.unibe.ch, christophe.g.lopez@gmail.com (C. Lopez).
} 


\section{Contents}

1. Introduction . . . . . . . . . . . . . . . . . . . . . . . . . . . . . 120

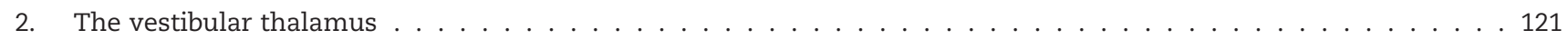

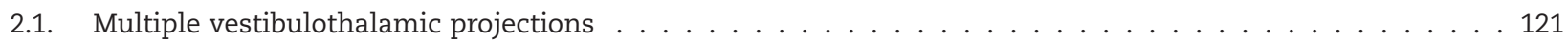

2.1.1. Vestibular projections to the ventroposterior complex . . . . . . . . . . . . . . . . . 121

2.1.2. Vestibular projections to the ventroanterior and ventrolateral nuclear complex . . . . . . . . . . . 123

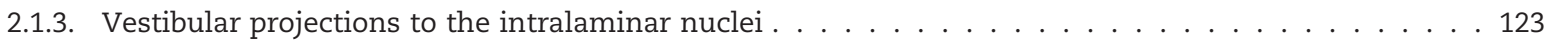

2.1.4. Vestibular projections to the thalamic posterior nuclear group . . . . . . . . . . . . . . . . . 123

2.1.5. Other vestibular thalamic nuclei . . . . . . . . . . . . . . . . . . . . . . . . . . . . 124

2.2. Functional organization of the vestibulothalamic pathways . . . . . . . . . . . . . . . . . . . 124

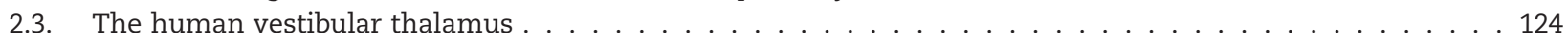

2.3.1. Neuroimaging data . . . . . . . . . . . . . . . . . . . . . . . . . . . 124

2.3.2. Neurological data . . . . . . . . . . . . . . . . . . . . . . . . . . 125

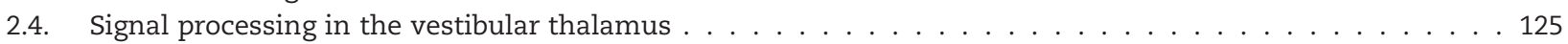

2.4.1. Sensory thalamic relay functions. . . . . . . . . . . . . . . . . . . . . . . . 125

2.4.2. Drivers and modulators in the vestibular thalamus. . . . . . . . . . . . . . . . . . . . . 126

2.4.3. Thalamic response to vestibular stimulation is very similar to that of vestibular nuclei . . . . . . 126

2.4.4. Responses to linear and gravitational acceleration . . . . . . . . . . . . . . . . . . . 127

2.4.5. Thalamic response to active and passive movements . . . . . . . . . . . . . . . . . . 127

2.4.6. Thalamic response to visual and somatosensory signals . . . . . . . . . . . . . . . . . . 128

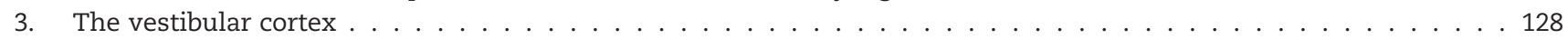

3.1. The "vestibular cortex" identified by electrophysiology in animals and by neuroimagery in humans . . . . . 128

3.1.1. Vestibular projections to the parietal cortex. . . . . . . . . . . . . . . . . . 128

3.1.2. Vestibular projections to the "parieto-insular vestibular cortex" and temporo-parietal junction . . . . 131

3.1.3. Vestibular projections to the frontal cortex . . . . . . . . . . . . . . . . . . . 133

3.1.4. Vestibular projections to the cingulate cortex . . . . . . . . . . . . . . . . . . . . 134

3.1.5. Vestibular projections to striate and extrastriate visual cortex. . . . . . . . . . . . . . . . . . . 134

3.1.6. Vestibular projections to the hippocampus . . . . . . . . . . . . . . . . . . . . . . . . . . 134

3.2. Connections between vestibular cortical areas . . . . . . . . . . . . . . . . . . . . . . . . 135

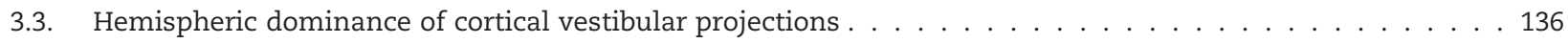

4. Human vestibular cortex in neurological disease . . . . . . . . . . . . . . . . . . . . . . . . 137

4.1. The search for human PIVC: electrical stimulation and brain damage to the insula and temporo-parietal junction . . 137

4.2. Electrical stimulation and brain damage to the parietal cortex . . . . . . . . . . . . . . . . . 138

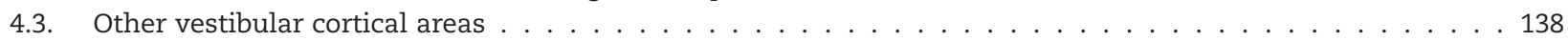

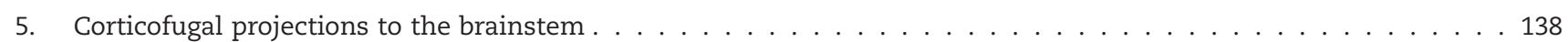

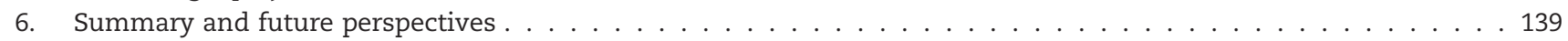

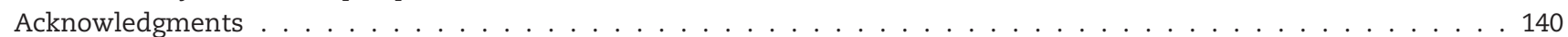

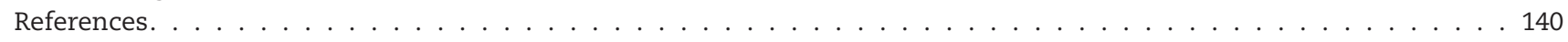

\section{Introduction}

The vestibular system has a unique role in the sensorimotor control and perception. By sensing the angular and linear accelerations, the vestibular system codes three dimensional head movements in space. By sensing gravitational acceleration, the vestibular receptors are also an essential basis for a spatial frame of reference allowing the brain to organize the erected human posture with respect to the ground (Berthoz, 2000). In turn, activation of the vestibular receptors is responsible for many reflexes acting on extraocular muscles devoted to gaze stabilization, as well as reflexes acting on postural muscles devoted to body orientation and stabilization in space (Wilson and Melvill Jones, 1979).

There is increasing evidence that the vestibular system is not only involved in perception, oculomotor and postural control, but also takes part in spatial cognition. In particular, how animals and human navigate in space - i.e. integrate and memorize the paths taken, elaborate and use cognitive maps of the spatial displacements - has been associated with vestibular processing (Berthoz et al., 1995; Mittelstaedt, 1999; Smith et al., 2010). In addition, vestibular signals, and the neural structures involved in vestibular processing, are crucial for distinguishing self-motion and object-motion (Straube and Brandt, 1987), perceiving the world as upright (Brandt and Dieterich, 1994; Lopez et al., 2007; Mittelstaedt, 1999), elaborating an internal model of gravity and of one's body motion (Angelaki et al., 2004; Merfeld et al., 1999), as well as for visual perception related to gravity (Indovina et al., 2005; Lopez et al., 2009). More recent studies conducted in neurological patients even suggested that vestibular signals are crucial for various aspects of one's body perception and awareness (Bottini et al., 1995; Vallar et al., 1993), and more generally for human bodily self-consciousness (Blanke et al., 2002; Lopez et al., 2008). 
Thus, the understanding of the neural correlates of these aspects of vestibular function (spatial perception and cognition, spatial navigation, and own body perception), as well as the understanding of the complex spatial and bodily symptoms observed in patients suffering from vestibular disorders, necessitate a clear description of the vestibular pathways to the thalamus and cerebral cortex. Yet, as compared to what is known about the central structures involved in visual, auditory and somatosensory processing, only a few comprehensive reviews have been written on the localization of the vestibular thalamus and cortex in the last 25 years (see Berthoz, 1996; Brandt and Dieterich, 1999; Fredrickson and Rubin, 1986; Fukushima, 1997; Guldin and Grüsser, 1998; for reviews up to 1999). Electrophysiological and tracer studies in animals have identified at least ten regions that are clearly involved in vestibular processing and that are widely distributed in parieto-insular, somatosensory, posterior parietal, and frontal cortices. As early as the 1990s, with the development of functional neuroimaging in human neuroscience, the number of studies aiming at determining the corresponding vestibular regions in the human brain has increased considerably. The variety of the techniques used for determining vestibular projections (cold and warm caloric vestibular stimulation, monaural or binaural galvanic vestibular stimulation, auditory stimulation) has led to a large body of functional and anatomical data, which has not been reviewed recently and systematically compared between animals and humans.

The aim of the present review is to summarize current knowledge on the anatomy of the vestibulothalamocortical system by establishing theoretical connections between data from animal electrophysiology and neuroanatomy with data from functional neuroimagery and neurology in humans. The latter include electrical stimulation of the cortex in epileptic patients and the description of patients with focal brain damage reporting vestibular dysfunctions. As the anatomy and physiology of the vestibular nuclei and cerebellum have been largely described in the literature (Angelaki and Cullen, 2008; Barmack, 2003; Berthoz and Vidal, 1993; Carpenter, 1988; Cullen et al., 2003; Lacour and Borel, 1993), the present review focuses on the identification of the multiple thalamic nuclei and cortical areas receiving vestibular inputs in animals and humans. Moreover, because the electrophysiological responses of single neurons in vestibular cortex have been reviewed in detail previously (Angelaki and Cullen, 2008; Berthoz, 1996; Fukushima, 1997; Grüsser et al., 1994; Guldin and Grüsser, 1998), they will not be discussed here.

\section{The vestibular thalamus}

As for the other sensory systems the thalamus relays and modulates the flow of information to the cortex (Behrens et al., 2003; Jones, 1985) and further plays a crucial function in corticothalamocortical pathways (Guillery and Sherman, 2002; Sherman and Guillery, 2002; Sherman, 2005). Second order neurons located in the vestibular nuclei project to thalamic nuclei, whose neurons process and relay sensory information to the cortex. Thalamic responses to vestibular stimulation have been recorded electrophysiologically as early as the 1970s in cats (Blum and Gilman, 1979; Sans et al., 1970) and monkeys (Büttner and Henn, 1976; Büttner et al., 1977; Deecke et al., 1974; Deecke et al., 1977; Magnin and Fuchs, 1977). In the same decade, vestibulothalamic pathways have been identified by various anatomical methods: degeneration techniques after electrolytic lesions of the vestibular nuclei (Raymond et al., 1974), injection of retrograde tracers into thalamic nuclei, and injection of anterograde tracers into the vestibular nuclei (Condé and Condé, 1978; Lang et al., 1979; Magnin and Kennedy, 1979). These pioneering studies, as well as more recent research (see Fukushima, 1997; Meng et al., 2007; Shiroyama et al., 1999, for reviews), have established the main characteristics of the vestibulothalamic pathways, which we summarize below.

\subsection{Multiple vestibulothalamic projections}

Vestibulothalamic projections terminate in various thalamic nuclei. As a general rule, the pattern of the vestibulothalamic pathways is consistent across species. A consistent pattern of vestibulothalamic projections is illustrated for New World monkeys (squirrel monkey) and old Word monkeys (Rhesus monkey) in Fig. 1A-B. Nevertheless, because of differences in thalamic anatomy across species and multiple nomenclatures (Jones, 1985; Percheron et al., 1996; Shiroyama et al., 1999), the correlation between animal and human thalamic nuclei receiving vestibular inputs remains somewhat problematic. Yet, on the basis of the recent mapping of the human thalamus and thalamo-cortical connections (Behrens et al., 2003; Morel et al., 1997), we will propose analogies between animal and human vestibulothalamocortical projections in Section 2.3.

\subsubsection{Vestibular projections to the ventroposterior complex} The ventral posterior lateral nucleus (VPL) and the nucleus ventralis intermedius (Vim, a transitional zone between the VPL and the ventrolateral thalamic nuclei, see Shiroyama et al., 1999) receive vestibular inputs (Blum et al., 1979b; Blum and Gilman, 1979; Büttner and Henn, 1976; Deecke et al., 1977; Kotchabhakdi et al., 1980; Lang et al., 1979; Liedgren et al., 1976; Magnin and Fuchs, 1977; Marlinski and McCrea, 2008a, 2008b; Matsuo et al., 1994, 1995, 1999; Meng et al., 2007; Nagata, 1986; Sans et al., 1970; Shiroyama et al., 1999) (Fig. 1A-B). In the rat, vestibular projections to the VPL originate mainly from the bilateral superior vestibular nucleus (SVN) and medial vestibular nucleus (MVN) via the medial longitudinal fasciculus, the ascending tract of Deiters and the superior cerebellar peduncle (Nagata, 1986). According to data from Shiroyama et al. (1999) in the rat, the Vim receives information mainly from the contralateral SVN, MVN and descending vestibular nucleus (DVN). The VPL-Vim is known to be a major relay of proprioceptive and cutaneous inputs to primary (areas 3a, $3 \mathrm{~b}$, and 1) and secondary somatosensory cortex in non-human and human primates (Behrens et al., 2003; see Jones, 1985, p. 325). In addition, these thalamic nuclei project to several vestibular areas described in cats, including the anterior suprasylvian (ASS) cortex and posterior cruciate region (Blum et al., 1979b). Recent electrophysiological studies in monkeys (Matsuzaki et al., 2004) suggest that the VPL also projects to the superior parietal lobule and intraparietal sulcus that have also been described as important vestibular cortical areas (see Section 3). In turn, the VPL receives cortical 
projections from layer 6 cells located in the primary (areas 3a, 3b, 1 and 2) and secondary somatosensory cortex (Jones, 1985).

In the squirrel monkey, vestibular-responsive neurons were found in several clusters within the ventral posterior (VP) thalamus, as illustrated in Fig. 1A (Marlinski and McCrea, 2008a). A cluster of vestibular neurons was "located within the midst of adjoining areas [...] recognized as the posterior region of the VP, an area of the [lateral pulvinar], and the superior part of the [medial geniculate nucleus, MGN]" (Marlinski and

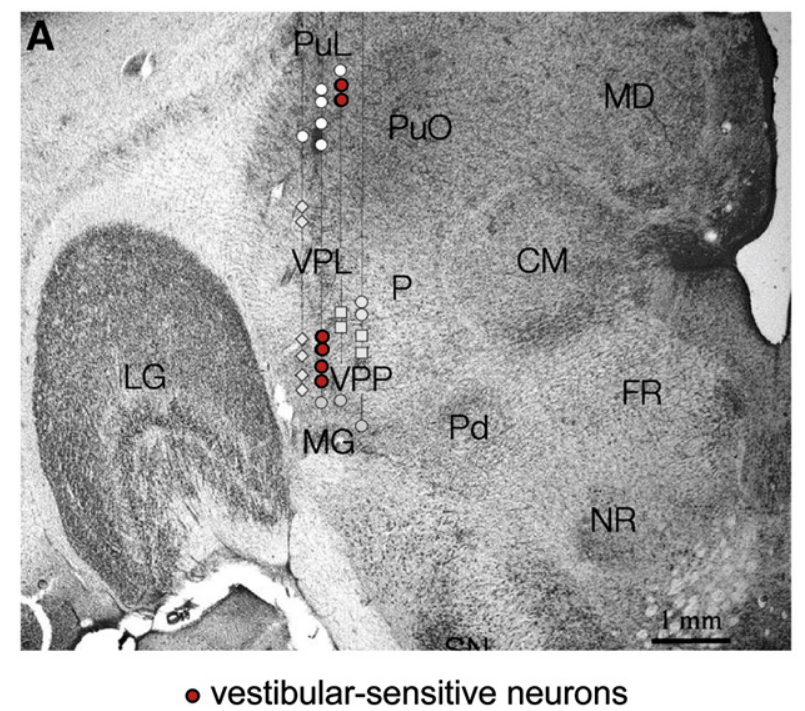

B
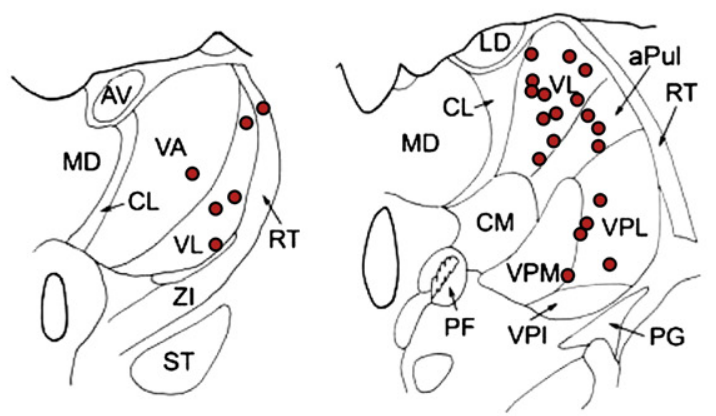

C

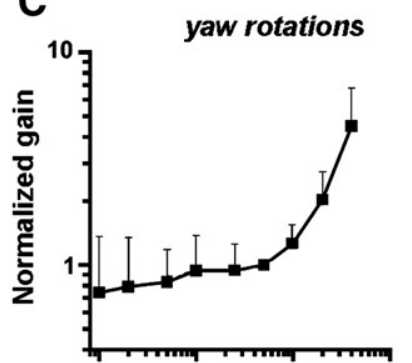

D
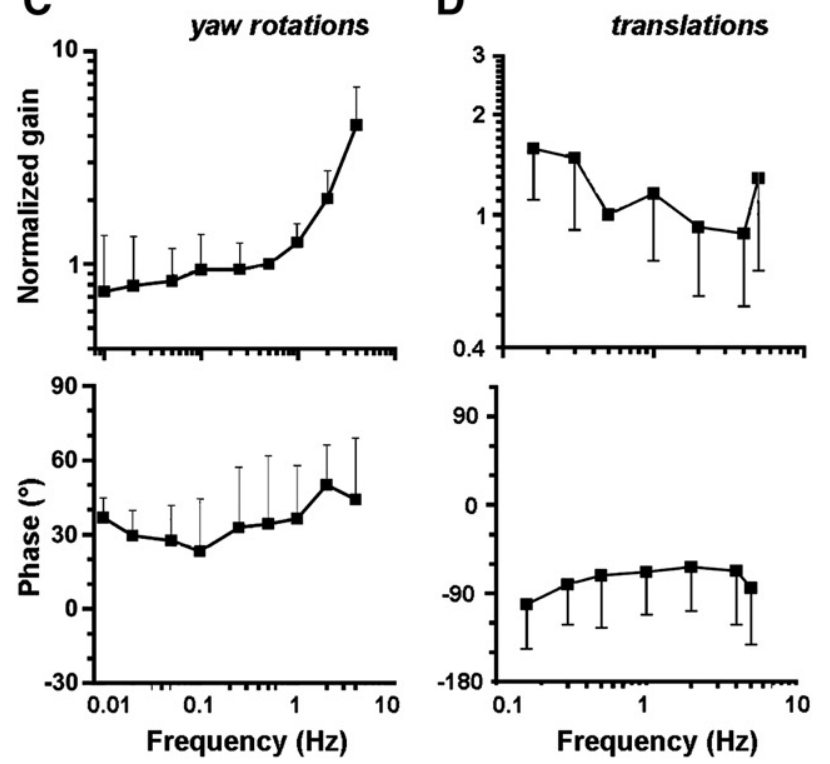

McCrea, 2008a). These authors suggested that this thalamic region may be the equivalent of the vestibular region described in the dorsal part of the MGN (Craig, 2004) and of the posterior part of the VP (VPP). The VPP was postulated to be the origin of the main thalamocortical pathway to the parietoinsular vestibular cortex (PIVC), the core vestibular region in the monkey (Akbarian et al., 1992). Finally, vestibular-sensitive neurons were also found in the superior border of the VP and may also project to the PIVC (Marlinski and McCrea, 2008a).

The ventral posterior inferior nucleus (VPI) includes neurons activated by rotatory vestibular stimulation (Büttner and Henn, 1976; Deecke et al., 1974, 1977; Magnin and Fuchs, 1977), as confirmed by autoradiographic studies in the macaque monkey (Lang et al., 1979). Previous experiments using tracers in the squirrel monkey indicate that the VPI is connected with the primary somatosensory cortex, mainly with the vestibular region in area $3 \mathrm{a}(3 \mathrm{aV})$ and area $3 \mathrm{~b}$, with posterior parietal cortex (areas 5 and 7), as well as the insula and the temporal cortex (Akbarian et al., 1992). Jones (1985, p.377) indicated that the VPI projects to the dysgranular field of the insular cortex.

Finally, the ventral posterior medial nucleus (VPM) can be considered as a vestibular thalamic nucleus. Anterograde tracing methods in the rat suggest that vestibular inputs originate from the MVN and SVN (Matesz et al., 2002). VPM neurons have been shown to respond to rotational and translational vestibular stimulation (Matsuo et al., 1994, 1995, 1999; Meng et al., 2007) as well as to electrical stimulation of the vestibular nerve (Blum et al., 1979b; Blum and Gilman, 1979; Deecke et al., 1977; Matsuo et al., 1994, 1999; Meng et al., 2001). However, Marlinski and McCrea (2008a), for example, found no or only few pure vestibular-sensitive neurons in the VPM of the squirrel monkey. Such differences could be related

Fig. 1 - Location of vestibular thalamic neurons in monkeys and their response to natural vestibular stimulation. The localization of vestibular-sensitive neurons (red circles) is represented on frontal sections of the thalamus in New World monkeys (A: squirrel monkey, Saimiri sciureus) and Old World monkeys (B: Rhesus monkeys, Macaca mulatta). aPul, anterior pulvinar nucleus; AV, anteroventral nucleus; CL, centrolateral nucleus; CM, central medial nucleus; FR, formatio reticularis; LD, lateral dorsal nucleus; LG, lateral geniculate body; MD, mediodorsal nucleus (nucleus medialis dorsalis); P, posterior nucleus; MG, medial geniculate body; NR, nucleus ruber; Pd, nucleus peripeduncularis; PF, parafascicular nucleus; PG, perigeniculate nucleus; PuL, pulvinar lateralis; PuO, pulvinar oralis; RT, reticular thalamic nucleus; ST, subthalamic nucleus; VA, ventroanterior nucleus; VL, ventrolateral nucleus; VPI, ventral posterior inferior nucleus; VPL, ventral posterior lateral nucleus; VPM, ventral posterior medial nucleus; VPP, nucleus ventroposterior, pars posterior; ZI, zona incerta. Adapted from Marlinski and McCrea (2008) for part A, and from Meng et al. (2007) for part B. Gain and phase of the responses of thalamic neurons to yaw rotations (C) and translations (D). Modified from Meng et al. (2007). 
to different delineation of this thalamic nucleus across species. In the Rhesus monkey, for example, Meng et al. (2007) proposed that the VPM activation could be equivalent to the VPI region previously identified by Deecke et al. (1974) in the same species. In addition to vestibular inputs, the VPM receives trigeminal inputs (see Jones, 1985, p. 339) and is connected to primary and secondary somatosensory cortex, predominantly with areas $3 b$ and 1 (Akbarian et al., 1992).

\subsubsection{Vestibular projections to the ventroanterior and} ventrolateral nuclear complex

Anatomical studies revealed vestibular projections to the ventroanterior thalamic nucleus (VA) and mostly to the ventrolateral thalamic nucleus (VL) [VA-VL complex] in the cat (Condé and Condé, 1978; Kotchabhakdi et al., 1980; Maciewicz et al., 1982; Nakano et al., 1985; Raymond et al., 1974, 1976; Sans et al., 1970), rat (Nagata, 1986; Shiroyama et al., 1999) and macaque monkey (Lang et al., 1979). Studies conducted in the cat and in the rat showed that the VA-VL complex receives vestibular afferents from the MVN and SVN via the medial longitudinal fasciculus, the ascending tract of Deiters and the superior cerebellar peduncle (Kotchabhakdi et al., 1980; Maciewicz et al., 1982; Nagata, 1986; Nakano et al., 1985; Shiroyama et al., 1999). Projections from the DVN and, to a lesser extent, from the lateral vestibular nucleus (LVN) have also been identified in the cat (Kotchabhakdi et al., 1980; Maciewicz et al., 1982). It has been demonstrated in the Rhesus monkey that the VL also receives vestibular afferents from the vestibular cerebellar nuclei (fastigial nucleus, dentate nucleus, anterior interposed nuclei; see Meng et al., 2007) (Fig. 1B). In addition, the VA is known to receive inputs mainly from the basal ganglia, and the VL mainly from the cerebellum (Percheron et al., 1996). The VA-VL complex is connected with the primary motor and the premotor cortex (Brodmann areas 4 and 6). Accordingly, this pathway may be considered as the major vestibulomotor pathway from the vestibular nuclei to premotor and motor structures.

\subsubsection{Vestibular projections to the intralaminar nuclei}

The intralaminar nuclei (IL) are a group of nuclei distributed in the internal medullary lamina of the thalamus and they have also been described as an important thalamic target of the vestibular nuclei. Regarding vestibular processing, anatomical studies have stressed the importance of the centromedian nucleus (or parafascicular nucleus in the rat), the central lateral nucleus, and the paracentral nucleus (Blum et al., 1979b; Blum and Gilman, 1979; Kotchabhakdi et al., 1980; Lai et al., 2000; Magnin and Kennedy, 1979; Matsuo et al., 1994; Meng et al., 2001, 2007; Nagata, 1986; Raymond et al., 1976; Shiroyama et al., 1995). The centromedian/ parafascicular nucleus receives vestibular information mainly from the MVN bilaterally, with an ipsilateral dominance, and from the SVN and the DVN ipsilaterally (Lai et al., 2000; Matesz et al., 2002; Shiroyama et al., 1995). The central lateral nucleus appears to receive many fibers only from the contralateral SVN, while the paracentral nucleus receives fibers mainly from the contralateral MVN and SVN, and bilaterally from the DVN (Kotchabhakdi et al., 1980). In turn, the IL nuclei project to the basal ganglia, where vestibular-evoked potentials have been recorded in the squirrel monkey (Liedgren and Schwarz, 1976). The IL nuclei have therefore been described as a major relay of the vestibulo-thalamo-striatal pathway, likely involved in the control of body and limb movements (Lai et al., 2000). In the rat, the parafascicular nucleus has connections with limbs and face regions of the somatic motor cortex (Aldes, 1988). In the squirrel monkey, the IL nuclei have also been described as a major vestibular relay to the PIVC, area $3 \mathrm{aV}$, and parietotemporal association area T3, some of the main areas of the vestibular cortex described in monkeys (Akbarian et al., 1992) (see Section 3).

\subsubsection{Vestibular projections to the thalamic posterior nuclear group}

The medial geniculate nucleus (MGN) contains neurons activated by electrical stimulation of the vestibular nerve (Blum et al., 1979a, 1979b; Matsuo et al., 1999; Roucoux-Hanus and BoisacqSchepens, 1977), as confirmed by anatomical studies showing vestibular projections to the MGN in the cat (Kotchabhakdi et al., 1980; Liedgren et al., 1976; Mergner et al., 1981) and in the rat (Nagata, 1986). The MGN receives projections mainly originating from the DVN (Kotchabhakdi et al., 1980; Matsuo et al., 1995; Mergner et al., 1981; Nagata, 1986), the SVN (Shiroyama et al., 1999) and the MVN (Kotchabhakdi et al., 1980; Mergner et al., 1981). In particular, the magnocellular division of the MGN, or MGmc (medial geniculate nucleus, pars magnocellularis), corresponding to the medial portion of the MGN and identified by its cellular morphology (Calford, 1983; Morest, 1964), has been involved in auditory, vestibular and somatosensory integration (Blum et al., 1979a). In the cat, the MGmc, together with other regions of the posterior group of the thalamus (PO), receives vestibular projections (Kotchabhakdi et al., 1980). In the squirrel monkey, Akbarian et al. (1992) proposed the MGmc to be the "caudal and inferior continuation of the "proprioceptive shell" of the ventroposterior thalamic complex", the VPP merging ventrally with the MGmc. Blum et al. (1979a) demonstrated a larger proportion of vestibular-responsive neurons in the MGmc than in the parvocellular part of the MGN and than in other PO regions. Older observations also revealed that lesions of the MGmc and PO suppressed vestibular-evoked responses in the cerebral cortex (Potegal et al., 1971; Spiegel et al., 1965). In addition to auditory afferents from the inferior colliculi and vestibular afferents from the vestibular nuclei, the MGmc receives afferents from the superior colliculi and the spinal cord, thus integrating multiple sensory signals (Blum et al., 1979a). The MGmc sends projections to the primary auditory cortex and to different sensory "association" areas surrounding the primary auditory cortex ("diffuse insular-temporal" cortical projections according to Jones, 1985; p. 92). In the squirrel monkey, the MGmc projects mainly to the vestibular granular insula (Akbarian et al., 1992) while in the cat it projects to the ASS cortex (Liedgren et al., 1976).

In the lateral geniculate nucleus (LGN) of the cat, Magnin and Putkonen (1978) recorded vestibular neurons responding to horizontal or vertical angular stimulation. Neurons activated by the stimulation of the posterior semicircular canals (pitch rotations) were found in the LGN (Matsuo et al., 1994). According to morphological studies, it seems, however, that the LGN receives only few vestibular projections, mainly originating from the contralateral MVN and DVN (Kotchabhakdi et al., 1980). The LGN is well known to be the main visual pathway from the retina to the primary visual cortex. The LGN is in turn innervated 
by the layer 6 of the visual cortices (areas 17, 18 and 19), and is therefore considered as a typical "first-order relay" passing visual information to the cerebral cortex (see Section 2.4. and for a review Sherman and Guillery, 2002).

Vestibular-sensitive neurons have also been described in the anterior and lateral pulvinar of squirrel and macaque monkeys (Marlinski and McCrea, 2008a, 2008b; Meng et al., 2007) (Fig. 1A-B). The pulvinar is a major integrator of visuomotor signals, afferenting extrastriate, temporal and posterior parietal cortices (Behrens et al., 2003; Jones, 1985). Anatomical work in the cat revealed projections from the pulvinar to layer 1 of areas 17 and 18, as well as to layer 4 of areas 19, 21a, and to the banks of the lateral and middle suprasylvian sulci (Sherman and Guillery, 2002). Tracer studies in squirrel monkeys indicate that the anterior and medial pulvinar are mainly connected to the vestibular area $3 \mathrm{aV}$, the lateral pulvinar to the PIVC and area T3, and that visual region of the lateral and inferior pulvinar are predominantly connected to area T3 (Akbarian et al., 1992). The pulvinar is in turn afferented by cortical layers 5 and 6 from several regions. Therefore, the pulvinar represents a "higherorder relay" processing and passing information from one cortical area to another, and modulating cortical and motor activity, thus differing from the "first-order relay" function of the LGN (see Section 2.4. and Sherman and Guillery, 2002).

Vestibular projections have also been demonstrated in the suprageniculate nucleus (SGN) of the rat (Shiroyama et al., 1999). These authors proposed that the SGN could be an important relay for vestibular information to the insula, frontal eye fields and parietal area 7 .

\subsubsection{Other vestibular thalamic nuclei}

In the rat, Shiroyama et al. (1999) described vestibular projections mainly from the MVN to the lateral dorsal nucleus (LD), a thalamic nucleus projecting to the visual cortex, area 7 and limbic system. Others also described vestibular projections to the lateral posterior nucleus (LP) in the cat (Liedgren et al., 1976; Matsuo et al., 1995), which is connected to the associative parietal areas 5 and 7 and to the ASS cortex (Liedgren et al., 1976).

\subsection{Functional organization of the vestibulothalamic pathways}

Given the multiple "vestibular" thalamic nuclei and the connections between specific vestibular nuclei and thalamic nuclei, specialized vestibulothalamic pathways subserving different functions have been hypothesized (Akbarian et al., 1992; Kotchabhakdi et al., 1980; Zwergal et al., 2009). There are, according to Kotchabhakdi et al. (1980), three main vestibulothalamic pathways in the cat, but their specific functions remain to be precisely determined. A first pathway originating from the MVN and DVN would project mainly to the MGN, the ventroposterior complex and VL, and is thus likely involved in vestibulo-somatosensory and motor functions. A second pathway originating from SVN would terminate in the IL nuclei and may be involved in vestibulo-striatal motor projections. The third pathway takes its origin in MVN, DVN, and SVN and terminates in the LGN. It could be involved in vestibulo-visual and visuo-motor functions. We note that the authors stressed the overlap between these pathways.
Although vestibulothalamic pathways are bilateral, most of anatomical studies have indicated that second order vestibular neurons project predominantly to the contralateral thalamic nuclei (Condé and Condé, 1978; Kotchabhakdi et al., 1980; Magnin and Kennedy, 1979). According to Lang et al. (1979) the laterality of the ascending projections of the vestibulothalamic pathways depends on the vestibular nuclei. Dominant ipsilateral vestibulothalamic projections have been shown for the LVN and MVN, whereas dominant contralateral projections have been found for the SVN. Additionally, the projection pattern may depend on the nature of the information driven by the vestibulothalamic neurons. Meng et al. (2001) showed that otolithic signals from utricular-activated vestibulothalamic neurons mostly terminate in the ipsilateral thalamic nuclei, while otolithic signals from saccular-activated vestibulothalamic neurons terminate in the contralateral thalamic nuclei. Electrophysiological recordings confirmed that most of VP neurons only sensitive to translation were activated by ipsilateral interaural translations (i.e., type I response, according to the classification of Duensing and Schaefer, 1958) (Marlinski and McCrea, 2008a). The semicircular projections have been reported to be mainly contralateral (Matsuo et al., 1994). These authors proposed that vestibular nuclei neurons responding to posterior semicircular canals activations send projections to the contralateral brainstem with several axonal branches making excitatory contacts in the thalamus (VPL, VPL, IL, VL, LGN) and oculomotor structures (oculomotor nuclei, interstitial nucleus of Cajal). The functional significance of this laterality remains however unclear.

\subsection{The human vestibular thalamus}

Data on the human vestibular thalamus are sparse. Several thalamic nuclei have been involved in vestibular processing on the basis of neuroimaging studies in healthy humans and neurological observations in epileptic patients and patients with focal thalamic lesions.

\subsubsection{Neuroimaging data}

There is currently only a very poor description of vestibular processing in the human thalamus based on neuroimaging techniques. This may be partly related to the deep location and small size of the thalamic nuclei, rendering difficult the parcellation of the thalamus into well-defined nuclei in the human brain when using neuroimaging. In addition, the relatively small number of neurons engaged in vestibular relay and processing, the fact that these neurons are organized in small patches distributed in the thalamus rather than in clearly identified nuclei (Meng et al., 2007), and the fact that complex processing occur in different populations of thalamic neurons involved in relaying or modulating the information (Guillery, 1995; Guillery and Sherman, 2002; Reichova and Sherman, 2004; Sherman and Guillery, 2002; Sherman, 2005), may have contributed to the limited description of the human vestibular thalamus. For other sensory modalities, successful delineation of the thalamus has been achieved in neuroimaging studies using higher magnetic field strengths (e.g. Chen et al., 1999), or in studies using diffusion tractography-based segmentation of the thalamus (e.g. Johansen-Berg et al., 2005) and resting-state functional connectivity 
(e.g. Zhang et al., 2008). No neuroimaging study using such methods has so far specifically focused on thalamic activations during vestibular stimulation. However, several neuroimaging studies describing the location of the vestibular cortex in healthy participants have also revealed thalamic activations during caloric (Bottini et al., 2001; Deutschländer et al., 2002; Dieterich et al., 2003; Marcelli et al., 2009; Suzuki et al., 2001) and galvanic (Bense et al., 2001; Bucher et al., 1998; Stephan et al., 2005) stimulation of the peripheral vestibular receptors. In a study using galvanic vestibular stimulation, Bense et al. (2001) reported an activation of the paramedian and dorsolateral thalamus, whereas in a study using caloric vestibular stimulation, Dieterich et al. (2003) reported an activation of the posterolateral and posteromedial thalamus. The activation of these two latter thalamic nuclei in humans is compatible with the implication of the ventroposterior complex of the monkey (VPL, VPI, VPM, VPP) in vestibular processing (Büttner and Henn, 1976; Deecke et al., 1974, 1977; Lang et al., 1979; Marlinski and McCrea, 2008a). Bucher et al. (1998) reported that galvanic vestibular stimulation evoked a bilateral activation of two thalamic regions: one located in the posterior median thalamus, and a second located in the anterior paramedian thalamus (that was also activated during a control nociceptive stimulation). These authors argued that the thalamic nuclei involved in vestibular processing were the nucleus medialis, nucleus habenularis and the pulvinar. As reported in animal studies (Marlinski and McCrea, 2008a, 2008b; Meng et al., 2007), the pulvinar thus appears to be also involved in vestibular processing in humans. More specifically, the medial pulvinar has been found activated during caloric vestibular stimulation, known as activating the semicircular canal, and during stimulation of the neck muscles (Bottini et al., 2001). Pulvinar activation has also been reported during optokinetic stimulation (Bucher et al., 1997; Dieterich et al., 1998), suggesting the importance of this thalamic nucleus for multisensory integration of vestibular, visual and proprioceptive signals.

Despite this evidence of multisensory convergence in some thalamic regions, the exact nature of the sensory processing taking place in the human vestibular thalamus is poorly understood. It is for example unknown whether attention and top-down mechanisms can influence vestibular processing through corticothalamic projections. The influence of corticothalamic feedback has already been demonstrated for the visual, auditory and somatosensory systems at the level of the LGN, MGN, and VPM (Alitto and Usrey, 2003; Ghazanfar et al., 2001; Sillito et al., 2006; Temereanca and Simons, 2004). An fMRI investigation in healthy participants revealed that attention directed to visual stimuli enhanced neural activity in the LGN, whereas it decreased neural responses to unattended visual stimuli (O'Connor et al., 2002). Attention was also effective in modifying the baseline activity within the LGN, despite any visual stimulation. Whether corticothalamic feedback influences vestibular thalamic activity and processing therefore deserves future investigations. This should be of relevance for human vestibular physiology because some behavioral studies have shown that top-down mechanisms can influence vestibular function (e.g. the gain of the vestibulo-ocular reflex (Melvill Jones et al., 1984)). This occurs presumably through corticofugal projections to the vestibular nuclei, but a similar influence could be expected from corticothalamic projections. Top-down mechanisms could also help distinguishing between self- and object-motion by acting on neural populations involved in vestibular coding only or combining vestibular, visual and proprioceptive signals.

\subsubsection{Neurological data}

Clinical data also suggest that the posterolateral thalamus (VPL/Vim) is a major relay station for vestibular signals (Dieterich et al., 2005a). Hawrylyshyn et al. (1978) were able to evoke in conscious patients sensations of movement by stimulating a vestibulothalamic pathway "situated ventral to the medial lemniscus, passing lateral to the red nucleus and dorsal to the subthalamic nucleus prior to terminating in the Vim." A more recent study using $\mathrm{H}_{2}^{15} \mathrm{O}$ PET measurements demonstrated projections of the Vim to the parieto-insular vestibular cortex (PIVC), the main vestibular cortical area (Ceballos-Baumann et al., 2001). In this study, electrical stimulation of the Vim decreased regional cerebral blood flow in vestibular regions in the contralateral retroinsular cortex in the depth of the superior temporal gyrus (area 22/42). Furthermore, it has been reported that patients with lesions of the posterolateral thalamus involving the VPL/Vim may present tilt of the perceived visual vertical (Dieterich and Brandt, 1993a) and suffer from postural instability known as "contraversive pushing" (Karnath et al., 2005). Therefore, by relaying vestibular cues, and particularly graviceptive cues, this thalamic region could be involved in encoding gravity and controlling body orientation in space. Finally, a contribution of the MGN has also been suggested in humans during neurosurgical explorations of the thalamus (Hawrylyshyn et al., 1978). Sensations of movements through space were evoked during the stimulation of a vestibulothalamic pathway "associated with the auditory pathway (lateral lemniscus and brachium of the inferior colliculus)" to the MGN.

Clinical descriptions of patients with focal brainstem lesions have also helped clarify the vestibular pathways to the cerebral cortex. Both contralateral and ipsilateral vestibulothalamic tracts convey vestibular signals from the vestibular nuclei to the cerebral cortex. A vestibulothalamic tract running contralaterally to the vestibular nuclei has been described within the medial longitudinal fascicle (Dieterich and Brandt, 1993b). Another vestibulothalamic tract, running ipsilaterally from the vestibular nuclei to the posterolateral thalamus, has recently been postulated to be adjacent to the medial lemniscus tract (Zwergal et al., 2008). Finally, a direct bilateral pathway running from the vestibular nuclei to the insular vestibular cortex, and bypassing the thalamic relays, has been hypothesized (Dieterich et al., 2005a).

\subsection{Signal processing in the vestibular thalamus}

In the following sections we will review what is known about the functional properties of vestibular thalamic nuclei.

\subsubsection{Sensory thalamic relay functions}

Often the term "thalamic relay functions" is used, but this may be misleading as it is now well-accepted that the thalamus does not only pass sensory signals from the brainstem and spinal cord to the cerebral cortex, but that the thalamus is 
involved in signal processing within complex cortico-thalamocortical loops (Alitto and Usrey, 2003; Sherman and Guillery, 2002; Sillito et al., 2006). The control exerted by corticothalamic projections originating from layer 5 cells, thalamic interneurons, the thalamic reticular nucleus and the parabrachial region on thalamic relay cells, points to the role of the thalamus in cortico-cortical communication as well as sensorimotor modulation (Sherman, 2005). In addition, the thalamus plays a fundamental role in the generation and synchronization of thalamic and thalamocortical rhythms related to states of arousal, allowing a modulation of the information transmitted to the cortex. The description of the electrophysiological properties of the relay neurons or of the control of tonic/burst firing modes of relay neurons are beyond the scope of the present review, but they can be found in previous reports (McCormick and Huguenard, 1992; McCormick and Bal, 1997; Sherman, 2001, 2005).

Of importance for the present review article, a useful distinction based on the type of input arriving at the thalamus has been introduced by Sherman and Guillery, who established anatomical and functional differences between "drivers" and "modulators" inputs (Guillery and Sherman, 2002; Sherman and Guillery, 1998, 2002; Sherman, 2005). Driver inputs were proposed to bring the signals via the thalamus, as for example the retinal input that is transmitted from the retina to the LGN and the visual cortex. Modulators are all other inputs that modulate the transmission of the driver inputs through the thalamus. Further, these authors proposed that thalamic relays could be classified into "first-order" and "higher-order" relays as a function of the origin of the driver input (Guillery, 1995). First-order relays receive a specific type of (sensory) driver input from the periphery and transmit these signals to the cerebral cortex. Prototypical first-order relays are neurons in the LGN with the driver input being the visual signals from the retina, the ventral portion of the MGN with the driver input being from the inferior colliculus, and the VP with the driver input being the somatosensory signals from the medial lemniscus. By contrast, higher-order relays receive driver inputs from neurons located in the layer 5 of a given cortex and transmit it to another cortex, or receive inputs from the parabrachial region. Higher-order relays are thus involved in cortico-thalamo-cortical loops and control the flow of information between various cortical regions. Such higherorder thalamic relays include for example the pulvinar and the MGmc.

\subsubsection{Drivers and modulators in the vestibular thalamus}

Whereas the distinction between drivers/modulators inputs and first-order/higher-order relays has been described for the visual (Guillery and Sherman, 2002), auditory (Lee and Sherman, 2010) and somatosensory (Reichova and Sherman, 2004) systems, much less is known about the vestibular thalamus.

Given the absence of a primary vestibular cortex (the equivalent of striate cortex for the visual system and of Heschl's gyrus for the auditory system), one may wonder whether thalamic nuclei containing vestibular-responsive neurons can be defined as first-order or higher-order thalamic relays. Yet, there is evidence of neurons in the VP nucleus responding only to vestibular stimulation, i.e. to rotations or translations of the animals in darkness, without concurrent somatosensory signals (Marlinski and McCrea, 2008a). Similar "vestibular-only" neurons have repeatedly been described in the vestibular nuclei (Cullen et al., 2003; Tomlinson and Robinson, 1984). The existence of such neurons in thalamic nuclei suggests the presence of first-order thalamic relays containing neurons in which signals are not mixed with visual and somatosensory signals. On the other hand, we also note the presence of vestibular-responsive neurons in several thalamic nuclei classically identified as higher-order relays such as the pulvinar and the MGmc (Sherman and Guillery, 2002; Sherman, 2005). The presence of vestibular-responsive neurons in both latter thalamic nuclei suggests that they may be involved in cortico-thalamo-cortical loops, the pulvinar receiving drivers and modulators inputs from neurons in the layers 5 and 6 of various cortical regions, respectively. However, most of this circuitry remains to be studied in more detail, as recently addressed in the visual, auditory and somatosensory systems (Guillery and Sherman, 2002; Reichova and Sherman, 2004; Sherman and Guillery, 2002; Sherman, 2005).

\subsubsection{Thalamic response to vestibular stimulation is very} similar to that of vestibular nuclei

Only few studies investigated thalamic responses to head movements, and most have focused on responses to head rotations in the yaw plane (Büttner and Henn, 1976; Büttner et al., 1977; Magnin and Fuchs, 1977; Magnin and Putkonen, 1978; Meng et al., 2007), more rarely in the pitch plane (Matsuo et al., 1995). A recent electrophysiological study by Meng et al. (2007) in macaque monkeys described the dynamics of vestibular neurons during roll, pitch and yaw head rotations, as well as during horizontal translations (Fig. 1C-D). As a general rule, responses of thalamic neurons to head rotation have dynamic characteristics very similar to those described in the vestibular nuclei in terms of directional preference, gain, phase and sensitivity to active versus passive head motion. These characteristics are detailed in the following paragraphs.

Similarly to vestibular nuclei neurons, thalamic neurons exhibit directional preference, as defined by the classification introduced by Duensing and Schaefer (1958). For yaw and roll rotations, type I neurons are activated (i.e. increased their discharge rate) during rotations to the side ipsilateral to the recording site, whereas type II neurons are activated during rotations to the side contralateral to the recording site. Type III neurons are activated during rotations ipsilateral and contralateral to the recording site. Most of the neurons responding to yaw rotations were of type II in the VP thalamus (Marlinski and McCrea, 2008b), as well as in the LGN and reticularis thalamic nucleus (Magnin and Putkonen, 1978).

The frequency discharge and gain of thalamic neurons increase as the head rotation frequency increases, with a reduced gain for very low stimulation frequencies (Fig. 1C) (Büttner et al., 1977; Magnin and Fuchs, 1977; Meng et al., 2007). Responses to animal rotations were very close to that found in the vestibular nuclei and in the PIVC (Liu et al., 2010). Regarding the thalamic responses to head translation (otolithic input), the gain of the neural responses decreases with the translation frequency (Fig. 1D). Meng et al. (2007) indicated that responses to translations in thalamic neurons differed from those recorded in vestibular nuclei "suggesting some 
form of additional processing." Finally, most of the responsive neurons were driven by both rotational and translational stimulations, and only few neurons responded selectively for rotation or translation (Meng et al., 2007).

\subsubsection{Responses to linear and gravitational acceleration}

Previous recordings showed that vestibular nuclei neurons use signals from both otoliths and semicircular canals to distinguish between head translations and head tilts with respect to gravity (Angelaki et al., 2004). Similar properties have been shown in the thalamus of the Rhesus monkey (Meng et al., 2007). Thus, neuronal populations were described that encoded translation only, head tilt only, or encoding both translation and head tilt with different amplitudes. Most of these thalamic neurons responded to both translation and head tilt, suggesting that they presented a convergence of signals from the otoliths and semicircular canals. Meng et al. (2007) even raised the question why no unambiguous signal (i.e. related only to head translation or head tilt) was observed in the thalamus, given that the disambiguation was solved at the vestibular nuclei level. These authors proposed that "although perception would greatly benefit from distinguishing translation from head orientation relative to gravity, the opposite is true for postural responses" and postulated that "the distributed coding of translation and net acceleration in the thalamus reflects the functional need to have both signals available to serve various cortical functions." Indeed, a recent investigation demonstrated similar responses properties in the monkey PIVC (Liu et al., 2010). Altogether, these data by Angelaki and colleagues indicate similar neural properties in vestibular nuclei, vestibular thalamus, and vestibular cortex regarding the disambiguation of the gravito-inertial forces.

\subsubsection{Thalamic response to active and passive movements}

There is evidence that neural processing in the thalamus helps distinguish between active/voluntary and passive/ involuntary head motion. At the level of vestibular nuclei neurons, electrophysiological recordings in monkeys revealed strongly modulated (decreased) responses during active and voluntary head movements as compared to passive head movements, although primary vestibular afferents reliably code for active head movements (Angelaki and Cullen, 2008; Cullen et al., 2003; McCrea et al., 1999; Roy and Cullen, 2004). These observations indicated that such vestibular nuclei signals are strongly modulated depending on the context of the movement and that efference copy signals can cancel the response of vestibular nuclei neurons during active motion (Angelaki and Cullen, 2008; Cullen et al., 2003). Marlinski and McCrea (2008b) investigated this issue and described neural responses of the VP thalamus in the squirrel monkey. These

\section{whole-body rotation}
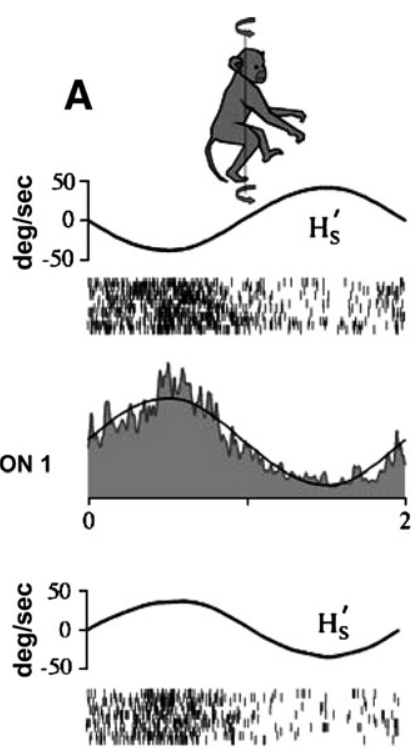

NEURON 2

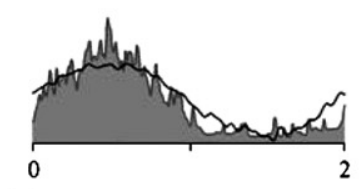

E
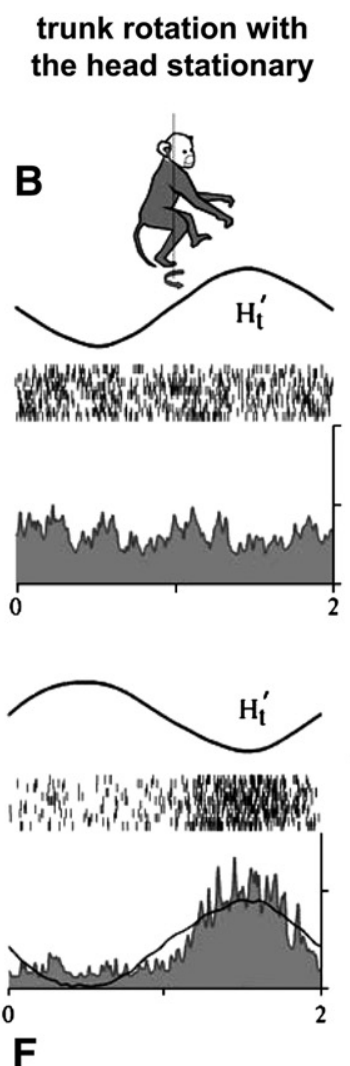

\section{involuntary head on trunk rotation}
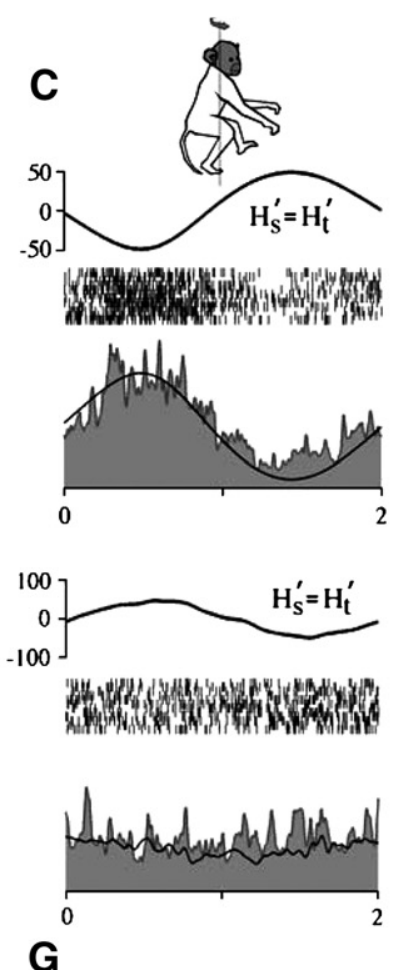

\section{voluntary head on trunk rotation}
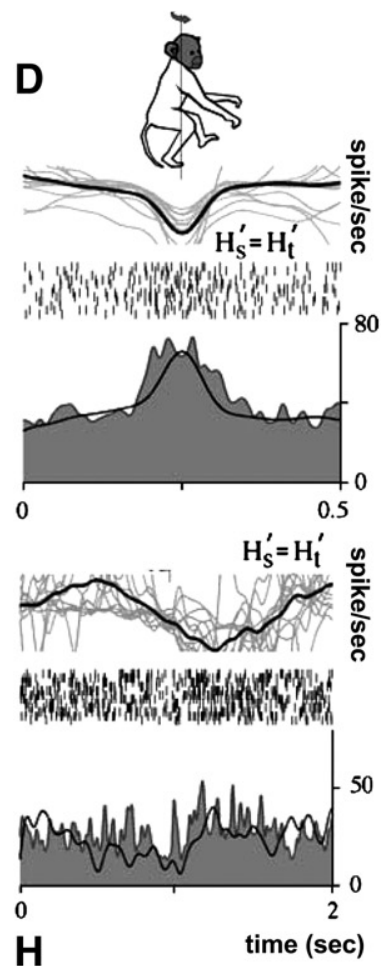

Fig. 2 - Response of ventral posterior thalamus neurons to active and passive head movements. Response of two neurons to voluntary and involuntary head movements and to trunk rotations under a stationary head. Neuron 1 is a typical "cephalokinetic neuron" and neuron 2 is a "somatokinetic neuron" (see text for details). The upper sinusoidal curve represents the angular velocity of the head relative to space $\left(\mathrm{H}_{\mathrm{s}}^{\prime}\right)$ or to the trunk $\left(\mathrm{H}_{\mathrm{t}}^{\prime}\right)$. The raster in the middle illustrates the spikes generated during the motion and the lower curve represents the mean firing rate. Adapted from Marlinski and McCrea (2008b). 
authors identified populations of neurons responding differently to whole-body rotation, proprioceptive neck stimulation and to voluntary and involuntary head rotations (Fig. 2). They found a population of "cephalokinetic neurons" that coded for the motion of the head in space with similar responses for whole-body motion (Fig. 2A) and head on trunk motion (Fig. 2C). These neurons did not respond to trunk rotation under a stationary head, and thus to proprioceptive stimulation (Fig. 2B). By contrast, "somatokinetic neurons" responded to both vestibular stimulation during whole-body rotation (Fig. 2E) and to rotation of the trunk under a stationary head through neck proprioception (Fig. 2F). Interestingly, the response of latter neurons to head on trunk motion was cancelled for both involuntary and voluntary movements (Fig. 2G-H), showing the influence of vestibular and neck proprioception for self-motion coding. Thus, the active/ passive head movement coding described previously at the level of vestibular nuclei (Angelaki and Cullen, 2008) is also a characteristic of thalamic neurons. Other studies indicated that similar self-motion coding may also take place in posterior parietal cortex of the macaque monkey (Klam and Graf, 2003; Klam and Graf, 2006), compatible with a modulator influence of the efference copy on VP neurons and corticothalamic projections to these vestibular thalamic neurons.

2.4.6. Thalamic response to visual and somatosensory signals Although vestibular-only neurons were recorded in the thalamus, some neurons integrating vestibular, visual and somatosensory signals have also been described in the thalamus. Vestibularly responsive neurons integrating optokinetic visual cues have been demonstrated in some thalamic nuclei of the Rhesus monkey (Büttner and Henn, 1976) and the cat (Magnin and Putkonen, 1978). Visual-vestibular interactions have been demonstrated in the LGN of the cat, where about half of the neurons responded to optokinetic stimulation (Magnin and Putkonen, 1978). Most of these neurons were also driven by saccadic eye movements. Electrophysiological recordings also showed somatosensory responses in thalamic neurons responding to vestibular stimulation. Neurons located in the VPL, VPM and VPI were shown to respond to deep proprioceptive signals from joints and muscles resulting from passive movements of the shoulder, leg, or vertebral column (Blum and Gilman, 1979; Büttner and Henn, 1976; Deecke et al., 1977). It seems that these vestibularly responsive neurons also respond to tactile input (Deecke et al., 1977), such as cutaneous stimulation applied to the anterior paw of the cat (Sans et al., 1970). Neurons with convergence of vestibular and neck proprioceptive signals have been shown to respond with a reciprocity in phase (e.g. for leftwards body rotation and rightwards trunk rotation, Fig. 2E-F) (Marlinski and McCrea, 2008b).

\section{The vestibular cortex}

Since the initial description of vestibular projections to the cat's cerebral cortex by Walzl and Mountcastle in 1949, multiple vestibular areas have been described in various species including humans. Fig. 3 summarizes the main vestibular areas in the monkey and human cerebral cortex. In Table 1 we give a detailed description of the human vestibular areas that have been identified by functional neuroimaging studies during caloric and galvanic stimulation of the peripheral vestibular apparatus. Vestibular-receiving areas are mostly distributed around the temporo-parietal junction (TPJ) and the posterior insula, the somatosensory cortex, posterior parietal cortex, anterior insula, lateral and medial frontal cortices. These areas constitute what has been called the "vestibular cortex" (Guldin and Grüsser, 1998).

\subsection{The "vestibular cortex" identified by electrophysiology in animals and by neuroimagery in humans}

\subsubsection{Vestibular projections to the parietal cortex}

\subsubsection{Projections to the anterior parietal cortex.}

Animal data. Vestibular projections to the primary somatosensory cortex have been shown in area $2 \mathrm{v}$ and area 3av. Early described as a region receiving vestibular inputs, area $2 \mathrm{v}$ was first thought to be the locus of the primary vestibular cortex in Rhesus monkey (Fig. 4A-B). Fredrickson et al. (1966) wrote that "the primary receiving area for the vestibular nerve is located in the posterior part of the postcentral gyrus at the base of the intraparietal sulcus between the first and second somatosensory fields, probably in Brodmann's area 2." Later studies showed that area $2 \mathrm{v}$ is located at the posterior border of area 2 , in the vicinity of areas 5 and 7, and posterior to areas devoted to somatosensory representations of the hand and the mouth (Fredrickson and Rubin, 1986; Schwarz and Fredrickson, 1971; Schwarz et al., 1973a, 1973b). Vestibular-evoked potentials recorded in area $2 \mathrm{v}$ of the monkey (Büttner and Buettner, 1978) and the cat (Jijiwa et al., 1991) indicated that this region receives inputs from the semicircular canals as well as the otoliths.

Two additional vestibular regions have been described at the level of the central sulcus in the so-called area $3 \mathrm{aV}$. "Area 3aHv" (for 3a-hand-vestibular region) is located in the somatosensory representation of the hand and arm in the squirrel monkey (Ödkvist et al., 1974) and the cat (Ödkvist et al., 1975). In the guinea pig, the vestibular region is located in the rostral part of the primary somatosensory representation of the forelimb (Ödkvist et al., 1973). "Area 3aNv" (for 3a-neckvestibular region) is located within the area coding for the neck and the trunk representations and extends more anteriorly into the primary motor cortex (Akbarian et al., 1994; Guldin et al., 1992; Guldin and Grüsser, 1998). According to Guldin and Grüsser (1998), about 30-50\% of 3aNv neurons receive vestibular inputs.

Human data. In keeping with electrophysiological data in animals, functional neuroimaging studies in humans revealed that vestibular stimulation led to activation of the intraparietal sulcus region (Fasold et al., 2002; Lobel et al., 1998; Suzuki et al., 2001). As activations were found in the anterior part of the intraparietal sulcus, at the junction of the intraparietal sulcus with the postcentral sulcus (Fasold et al., 2002; Lobel et al., 1998), we propose that this region may be homologous to the cat's and monkey's area 2v. Human neuroimaging studies also revealed activation of the primary somatosensory cortex, that could represent the human homologue of monkey's area 3aHv and/or 3aNv (Bottini et al., 1994; Emri et al., 2003; Fasold et al., 2002; Petit and Beauchamp, 2003). More work is needed 
A

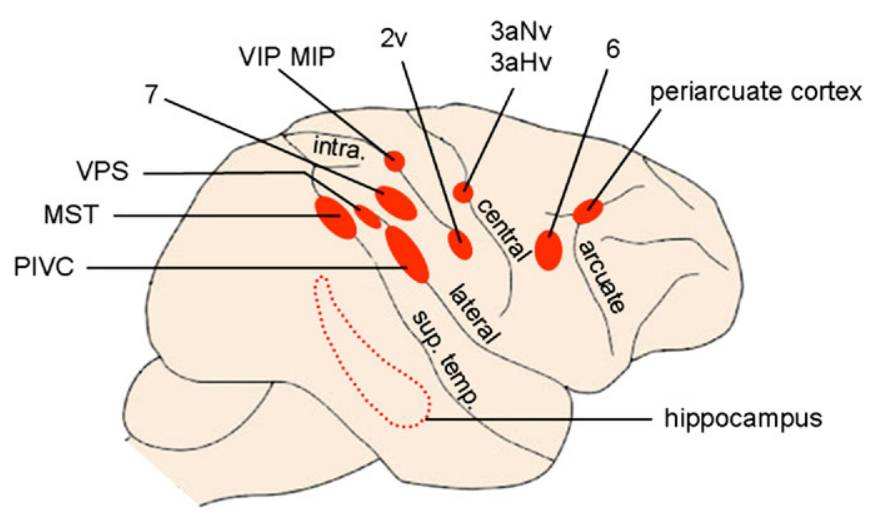

B

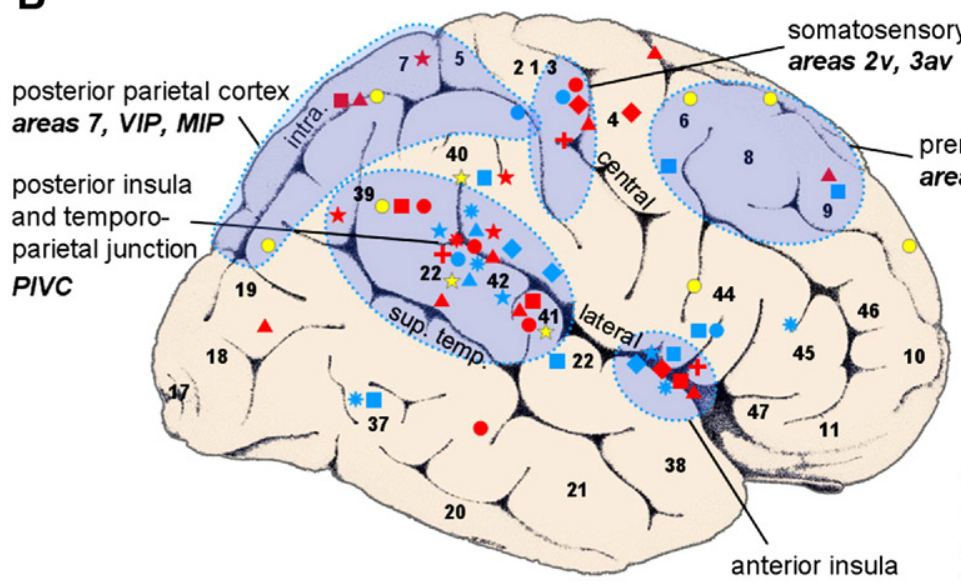

Bottini et al., 1994 $\star$ Vitte et al., 1996 Suzuki et al., 2001 A Fasold et al., 2002 Emri et al., 2002

* Hegemann et al., 2003

+ Indovina et al., 2005

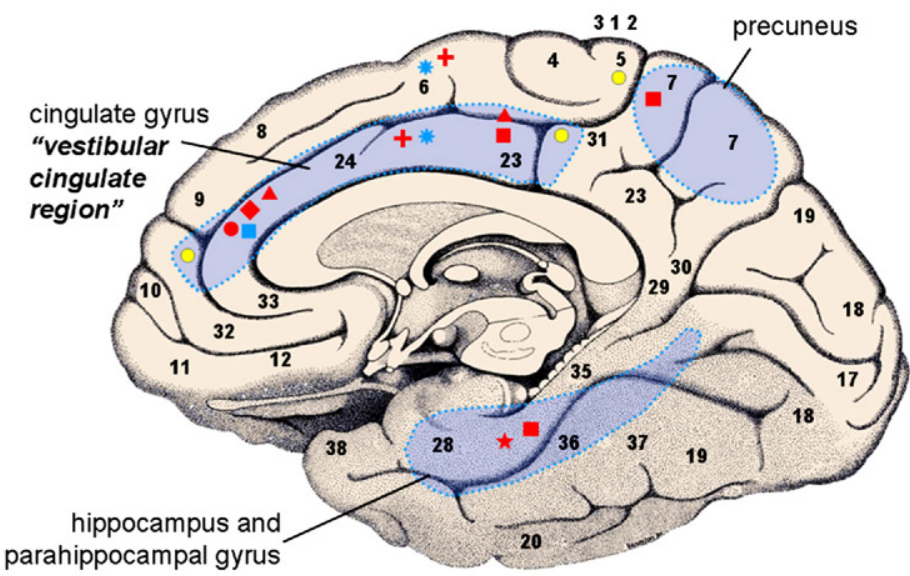

Lobel et al., 1998

$\star$ Bucher et al., 1998

Bense et al., 2001

$\triangle$ Fink et al., 2003

Eickhoff et al., 2006

* Stephan et al., 2005

- Miyamoto et al., 2007

ז Schlindwein et al., 2007

Fig. 3 - Comparative anatomy of monkey and human vestibular cortex. (A) Schematic representation of the vestibular areas in monkeys. Areas 2v, 6v, 7 and 3av (3aHv: 3a-hand-vestibular region, 3aNv: 3a-neck-vestibular region), MIP: medial intraparietal area, MST: medial superior temporal area, PIVC: parieto-insular vestibular cortex, VIP: ventral intraparietal area, VPS: visual posterior sylvian area. Major sulci are represented: arcuate sulcus (arcuate), central sulcus (central), lateral sulcus (lateral), intraparietal sulcus (intra.) and superior temporal sulcus (sup. temp.). Adapted from Sugiuchi et al., 2005. (B) Vestibular areas in humans revealed by neuroimagery during caloric (red symbols) and galvanic (blue symbols) vestibular stimulation, as well as during short auditory stimulation (yellow symbols). To summarize, right and left cerebral activations are reported on a lateral view of the right hemisphere (modified after Duvernoy, 1999). The supposed homologous vestibular areas reported in animals are indicated in bold letters (FEF: frontal eye fields). The numbers on the cortex refer to the cytoarchitectonic areas defined by Brodmann. Adapted from Lopez et al. (2008). 
Table 1 - Cortical and thalamic regions responding to artificial vestibular stimulation identified by neuroimagery (fMRI, PET, MEG).

Caloric vestibular stimulation

Galvanic vestibular stimulation

\begin{tabular}{|c|c|}
\hline Parietal cortex & Parietal cortex \\
\hline$\oplus$ Intraparietal sulcus ${ }^{[1,2]}$ & $\oplus$ Intraparietal sulcus ${ }^{[6]}$ \\
\hline $\begin{array}{l}\text { (Inferior parietal lobule: supramarginal gyrus BA } 40^{[2,4,3,10,15,17]} \text {, angular gyrus } \\
\text { BA } 39^{[3,15]}\end{array}$ & $\begin{array}{l}\oplus \text { Inferior parietal lobule }{ }^{[14]} \text {,supramarginal gyrus BA } 40^{[7,9,]} \\
\oplus \text { Postcentral gyrus }{ }^{[6]}\end{array}$ \\
\hline$\oplus$ Postcentral gyrus ${ }^{[15,17]}$ BA $3 / 1 / 2^{[4,5]}$ & $\oplus$ Middle cingulate gyrus BA $23^{[9]}$ \\
\hline$\oplus$ Superior parietal lobule $\mathrm{BA} 7^{[3]}$ and paracentral lobulus/precuneus $\mathrm{BA} 7^{[2,15]}$ & $\oplus$ Posterior parietal operculum ${ }^{[14]}$ \\
\hline$\oplus$ Posterior cingulate sulcus/cortex ${ }^{[1,10,12]}$, retrosplenial cortex ${ }^{[3]}$ & $\Theta$ Precuneus BA $7 / 23^{[7,9,]}$ \\
\hline$\Theta$ Precuneus BA $7^{[10]}$ & $\Theta$ Posterior cingulum ${ }^{[9]}$ \\
\hline Temporal cortex & Temporal cortex \\
\hline$\oplus$ Superior temporal gyrus ${ }^{[1,17]}$ BA $22 / 42^{[2,4,10,15]}$ BA $38^{[2,10]}$ & $\oplus$ Superior temporal gyrus ${ }^{[11]}$ BA $22^{[7,9,]}$ \\
\hline$\oplus$ Transverse temporal gyrus BA $21^{[4,10]}, \mathrm{BA} 41^{[1,3,4]}, \mathrm{BA} 42^{[3,10]}$ & $\oplus$ Transverse temporal gyrus ${ }^{[8]}$ \\
\hline$\oplus$ Superior and inferior temporal sulcus ${ }^{[1]}$ & $\oplus$ Middle temporal gyrus BA $37 \mathrm{MT} / \mathrm{V} 5^{[7,9]}$ \\
\hline$\oplus$ Hippocampus ${ }^{[2,3,15]}$ and parahippocampal gyrus BA $36 / 37^{[2]}$ & $\oplus$ Inferior temporal gyrus BA $37^{[9]}$ \\
\hline$\Theta$ Parahippocampal gyrus ${ }^{[9,10]}$, hippocampal gyrus ${ }^{[12]}$ & $\oplus$ Posterior lateral sulcus ${ }^{[6]}$ \\
\hline$\Theta$ Middle temporal gyrus BA 19/37 ${ }^{[10]} \mathrm{V} 5+/ \mathrm{MT}+{ }^{[12]}$ & $\begin{array}{l}\Theta \text { Hippocampus }{ }^{[9]} \text { and parahippocampal gyrus }{ }^{[9,]} \\
\Theta \text { Middle and inferior temporal gyrus BA } 21 / 20^{[9]}\end{array}$ \\
\hline Insular cortex & Insular cortex \\
\hline$\oplus$ Insular gyrus ${ }^{[2,3,5,13]}$ & $\oplus$ Insular gyrus $^{[7]}$ \\
\hline$\oplus$ Posterior insula ${ }^{[3,4,5,12,15,16,17]}$ & $\oplus$ Posterior insula ${ }^{[8,11,9,]} / \mathrm{BA} 40$ and transverse temporal gyrus ${ }^{[8]}$ \\
\hline$\oplus$ Anterior insula ${ }^{[1,12,15]}$ & $\oplus$ Anterior insula $^{[7,14,9]}$ \\
\hline$\oplus$ Parieto-insular cortex ${ }^{[1]}$ & $\oplus$ Middle insula and BA $22^{[8]}$ \\
\hline Frontal cortex & Frontal cortex \\
\hline$\oplus$ Central sulcus $^{[1]}$ & $\oplus$ Central sulcus ${ }^{[6]}$ \\
\hline$\oplus$ Precentral gyrus $[1,5,12,17]$ & $\oplus$ Precentral gyrus ${ }^{[6]}$ BA $6 /$ frontal eye fields ${ }^{[7,9]}$ Supplementary motor area ${ }^{[9]}$ \\
\hline$\oplus$ Inferior frontal gyrus ${ }^{[1,12,17]} \mathrm{BA} 46 / 44^{[15]}$ & $\oplus$ Inferior frontal gyrus ${ }^{[6]} \mathrm{BA} 44 / 47^{[7,9]} \mathrm{BA} 45^{[9]}$ \\
\hline$\oplus$ Middle frontal gyrus ${ }^{[15,17]}$ & $\oplus$ Middle frontal gyrus ${ }^{\left[{ }^{[6]}\right.} \mathrm{BA} 9 / 46^{[7,9,]}$ \\
\hline$\oplus$ Superior frontal gyrus BA $6 / 8 / 10^{[15]}$, supplementary motor area ${ }^{[17]}$ & $\oplus$ Anterior cingulate gyrus BA $32^{[7]}$ \\
\hline$\oplus$ Precentral sulcus ${ }^{[1]}$ & $\Theta$ Transuerse frontopolar gyrus ${ }^{[6]}$ \\
\hline$\oplus$ Dorsolateral prefrontal cortex ${ }^{[1]}$ & $\Theta$ Central sulcus BA $4^{[7]}$ pre-/postcentral gyrus BA $6 / 4 / 3^{[7,9]}$ \\
\hline$\oplus$ Anterior cingulate gyrus ${ }^{[1,5,10]}$ BA $24^{[2,4]}$ BA $32^{[15]}$, middle cingulate gyrus ${ }^{[17]}$ & $\Theta$ Middle and superior frontal gyrus BA $9 / 10^{[9]}$ \\
\hline \multirow{2}{*}{\multicolumn{2}{|c|}{$\Theta$ Superior dorsal and middle frontal gyrus ${ }^{[12]}$}} \\
\hline & \\
\hline Occipital cortex & Occipital cortex \\
\hline$\oplus$ Occipital lateral gyrus ${ }^{[1]}$ & $\Theta$ Inferior and superior occipital gyrus BA $17 / 18 / 19^{[7,9]}$, cuneus ${ }^{[7,9]}$, \\
\hline$\Theta$ Visual cortex BA $17 / 18 / 19^{[10]}$, calcarine cortex V1/V2 ${ }^{[12]}$, cuneus ${ }^{[12]}$ & calcarine sulcus ${ }^{[9]}$ \\
\hline$\Theta$ Inferior, middle and superior occipital gyrus ${ }^{[12]}$ & $\Theta$ Fusiform gyrus BA $18^{[7,9]}$ \\
\hline \multirow[t]{2}{*}{$\Theta$ Fusiform gyrus ${ }^{[12]}$} & $\Theta$ Middle occipital gyrus BA 18/19 [9] \\
\hline & Thalamus \\
\hline $\begin{array}{l}\text { Thalamus } \\
\oplus \text { Thalamus }^{[2,10,12,13,17]} \text { posterolateral/posteromedial }^{5]}\end{array}$ & $\oplus$ Thalamus $^{[8,9,]}$ paramedian and dorsolateral ${ }^{[7]}$ \\
\hline
\end{tabular}

Caloric vestibular stimulation refers to irrigation of the external auditory canal with cold or warm water, air or gas. Galvanic vestibular stimulation refers to monaural or binaural electrical stimulation applied to the mastoid processes. The symbols $\oplus$ and $\Theta$ refer, respectively, to an increase and decrease of BOLD signal or regional cerebral blood flow. BA: Brodmann area. According to the data from: [1] Fasold et al. (2002); [2] Suzuki et al. (2001); [3] Vitte et al. (1996); [4] Bottini et al. (1994); [5] Emri et al. (2003); [6] Lobel et al. (1998); [7] Bense et al. (2001); [8] Bucher et al. (1998); [9] Stephan et al., 2005; [10] Deutschländer et al., 2002; [11] Fink et al. (2003); [12] Bottini et al. (2001); [13] Marcelli et al. (2009); [14] Eickhoff et al., 2006b; [15] Dieterich et al. (2003); [16] Hegemann et al., 2003; [17] Indovina et al., 2005.

to localize these three vestibular regions in humans anatomically and functionally (see Blanke et al. (2000a)).

\subsubsection{Projections to the posterior parietal cortex.}

Animal data. A vestibular contribution of area 7 has been shown in monkeys (Faugier-Grimaud and Ventre, 1989; Leinonen et al., 1980), although Guldin and Grüsser (1998) proposed that area 7 should not be considered a part of the vestibular cortex, given that only a low percentage of area 7 neurons were driven by vestibular inputs.
Vestibular neurons have also been recorded in the fundus of the intraparietal sulcus, in the ventral intraparietal area (VIP) and the medial intraparietal area (MIP) (Bremmer et al., 2001, 2002; Klam and Graf, 2003, 2006; Schlack et al., 2002). Area VIP is a multimodal area important for three-dimensional spatial coding (Schlack et al., 2005). This area receives visual inputs from the middle temporal area (MT) and the medial superior temporal area (MST) (Colby et al., 1993), and is strongly interconnected with the motor cortex (Matelli and Luppino, 2001). According to Bremmer et al. (2002), about one third of 
A

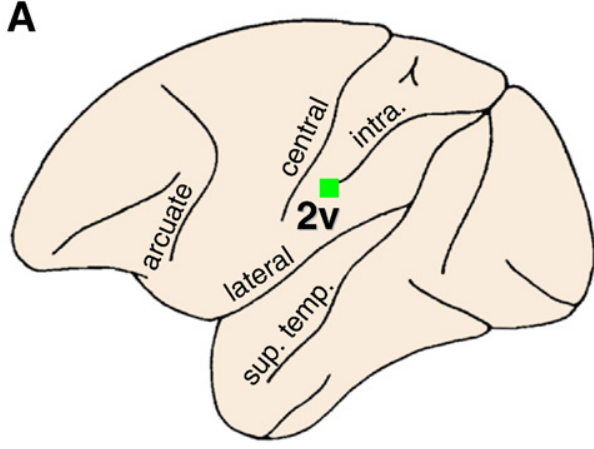

B

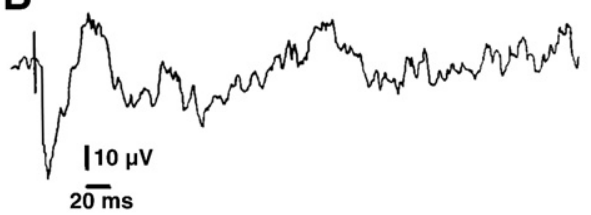

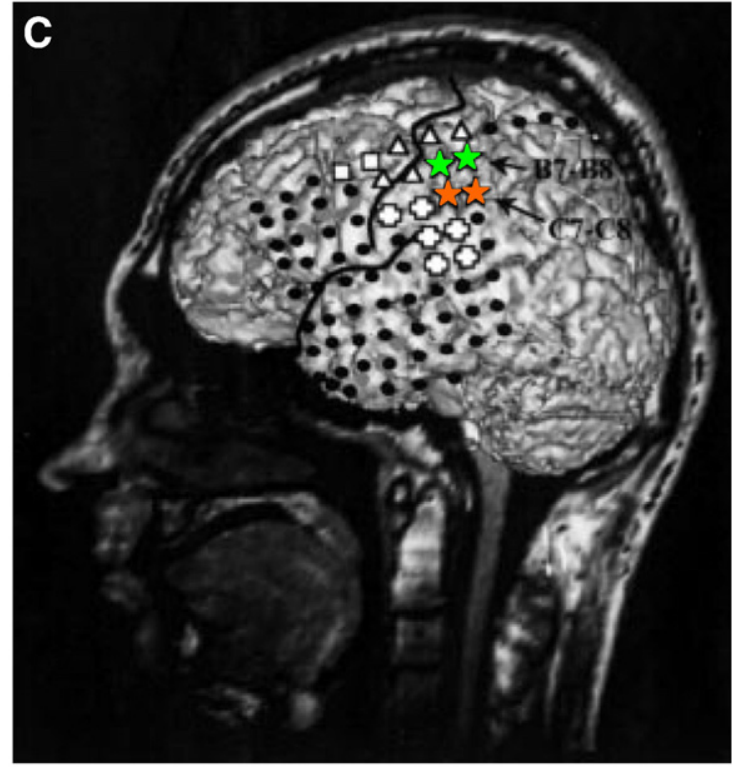

Fig. 4 - Vestibular area $2 v$ in monkey and human. (A) Localization of area $2 v$ in the Rhesus monkey (the image has been flipped horizontally to match orientation of C). The green square at the tip of the intraparietal sulcus represents the site at which evoked potentials were recorded during electrical stimulation of the ipsilateral vestibular nerve (an average vestibular evoked-potential with a 5-6 ms latency is illustrated in B). Adapted from Fredrickson et al. (1966). (C) Vestibular responses during electrical stimulation of the parietal cortex in an epileptic patient. Electrical cortical stimulation at electrodes B7-B8 (green stars) evoked sensations of vertical linear and rotational body motion. Stimulation at electrodes C7-C8 (orange stars) evoked sensations of an alternating right-left whole body motion. The uppermost black line represents the central sulcus and the black line located below represents the lateral sulcus. Reproduced from Blanke et al. (2000).

neurons in area VIP are sensitive to rotations around the vertical axis.

Human data. Vestibular stimulation has been shown to activate the inferior parietal lobule, at the level of the angular gyrus (area 39) and supramarginal gyrus (area 40), and such foci may represent the human homologue of monkey area 7 (Bense et al., 2001; Bottini et al., 1994, 1995; Dieterich et al., 2003; Schneider et al., 2006; Suzuki et al., 2001; Vitte et al., 1996). The inferior parietal lobule has also been shown to be activated by head movements in space and changes in gaze direction (Petit and Beauchamp, 2003).

Human neuroimaging studies have also revealed intraparietal sulcus activation (Fasold et al., 2002; Lobel et al., 1998; Suzuki et al., 2001). An activation of the posterior intraparietal sulcus has been revealed by Miyamoto et al. (2007) during otolithic stimulation, and by Suzuki et al. (2001) during semicircular canals, suggesting a vestibular region in humans that is distinct from the more anterior human homologue of area $2 \mathrm{v}$. Finally, vestibular stimulation also activated the lateral superior parietal lobule (human area 7) (Vitte et al., 1996), and medially the precuneus (Suzuki et al., 2001).

3.1.2. Vestibular projections to the "parieto-insular vestibular cortex" and temporo-parietal junction

Animal data. Grüsser and colleagues were the first to describe vestibularly-driven neurons in macaque, squirrel and marmoset monkeys in a region they called "parieto-insular vestibular cortex," or PIVC (Grüsser et al., 1982, 1990a, 1990b, 1994; Guldin et al., 1992, 1993; Guldin and Grüsser, 1998). The location of PIVC in the Java monkey, Macaca fascicularis, is illustrated in Fig. 5A. According to these authors, vestibular neurons were located "in the upper bank of the lateral sulcus around the posterior end of the insula, sometimes also within the upper posterior end of the insula [... and] more posteriorly in the retroinsular region or more anteriorly in the parietal operculum." Yet, inter-species differences have been described regarding the location of the PIVC. Whereas PIVC is located on the parietal lip of the lateral sulcus in Old Word monkeys (macaque), it is located on the temporal lip of the lateral sulcus in New World monkeys (squirrel monkey). Cytoarchitectonically, the PIVC region is mainly part of the retroinsular areas $\mathrm{Ri}$ (area retroinsularis) or reipt (area retroinsularis pars parietalis) and of the granular insula (Ig, insula granularis) [see Augustine (1996), for a review of insula anatomy]. A part of the PIVC may be homologous to the ASS cortex of the cat where vestibular responses have also been recorded (Kempinsky, 1951; Liedgren et al., 1976; Mergner et al., 1985; Mickle and Ades, 1954; Mills and Taylor, 1974; Walzl and Mountcastle, 1949). More recently, Angelaki and colleagues provided an extensive description of the localization and responses of PIVC neurons in the Rhesus monkey, Macaca mulatta, using both MRI and electrophysiological recordings (Chen et al., 2010; Liu et al., 2010) (Fig. 5B). They found that vestibular neurons were mostly localized in "area $\mathrm{Ri}$ and in the transition zones between S2, Ri and Ig" (Chen et al., 2010). Finally, we note that posterior to the PIVC, Guldin and Grüsser (1998) also recorded vestibularly-driven neurons in the "visual posterior sylvian" (VPS) area. Although essentially driven by visual stimuli such as optic flow, this area has been 
A Java monkeys (Macaca fascicularis)

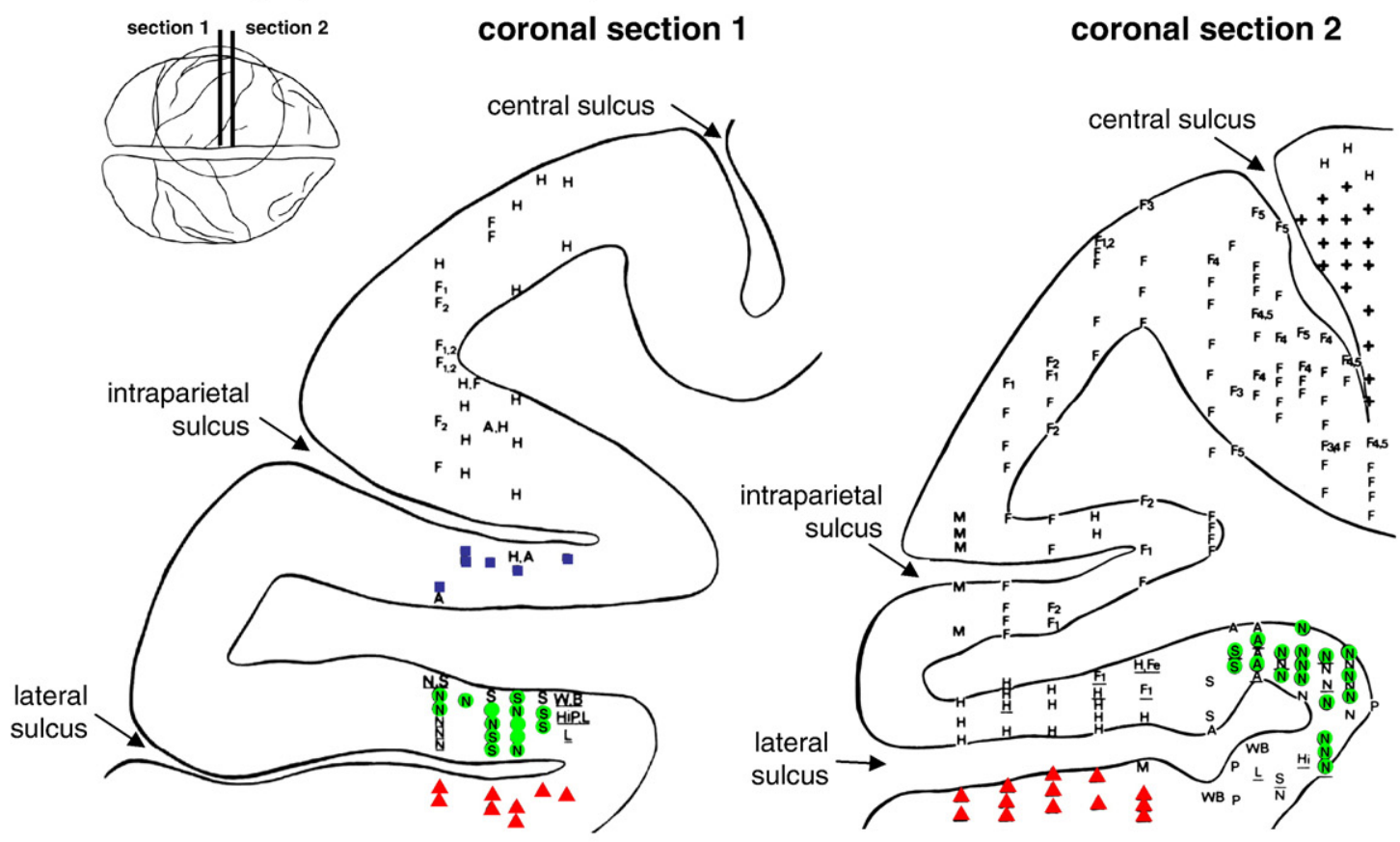

- vestibular response

+ motor response

$\Delta$ auditory response

- visual response somatosensory responses:

$\begin{array}{lll}\text { A, arm } & \text { C, chest } & \text { P, pelvis } \\ \text { Fe, feet } & \text { Fa, face } & \text { S, shoulder } \\ \text { F1-5, finger 1-5 } & \text { Hi, hip } & \text { WB, whole body } \\ \text { H, hand } & \text { L, leg } & \text { T, tail } \\ \text { H, contralateral } & \text { M, mouth } & \text { V, vertebra } \\ \text { H, bilateral } & \text { N, neck } & \end{array}$

B Rhesus monkeys (Macaca mulatta)

C Human

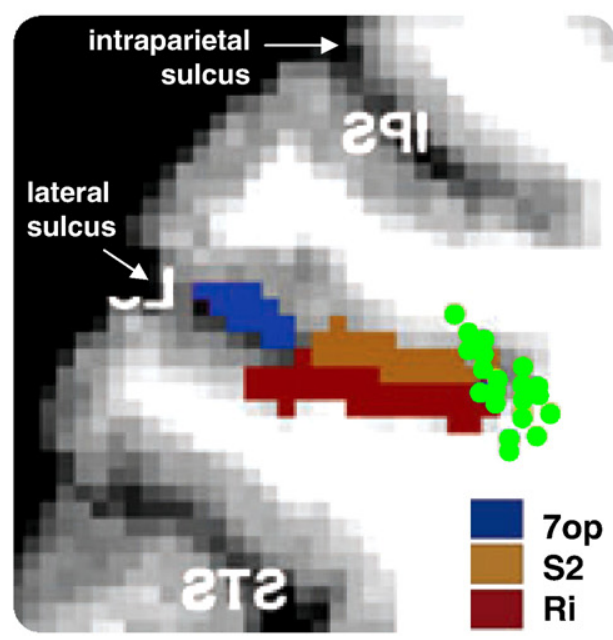

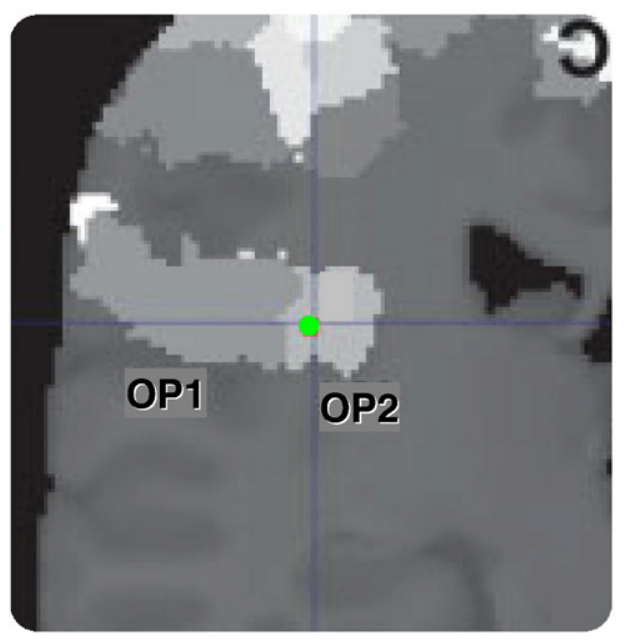

Fig. 5 - Comparative localization of the parieto-insular vestibular cortex (PIVC) in monkeys and humans. (A) The location of vestibular-responsive neurons (green dots) is represented on coronal sections of two Java monkeys. Adapted from Grüsser et al. (1990a). (B) Neurons responding to vestibular stimulation (green dots) are localized on an MRI scan of the brain of one Rhesus monkey. The colored areas represent the cytoarchitectonic areas 7op (area 7 operculum), Ri (retroinsular area) and S2 (secondary somatosensory area). The image has been flipped horizontally to show the similarity with part A. Adapted from Liu et al. (2010). (C) Galvanic vestibular stimulation activated the right parietal operculum (green dot, cytoarchitectonic area OP 2) at the junction of the posterior parietal operculum with the insular/retroinsular cortex. The image has been flipped horizontally to show the similarity with parts A and B. Adapted from Eickhoff et al. (2006b). 
reported to contain about $30 \%$ of vestibular-activated neurons.

In some of the PIVC neurons, Grüsser et al. reported a convergence of vestibular, somatosensory and visual signals (Grüsser et al., 1990a, 1994). PIVC neurons were not sensitive to the illumination of the room or the displacement of small visual objects, but were particularly sensitive to the displacement of structured patterns of more than $30^{\circ}$ (optokinetic stimulation) in a specific direction (Grüsser et al., 1990a). Most of PIVC neurons also had tactile receptive fields located in the region of the neck and responded to muscle pressure, vibrations, and to rotation of the neck. Grüsser et al. (1990a) reported that the most effective somatosensory stimulation that was found to activate PIVC neurons consisted of trunk rotations under a stable head (fixed in space). Neural responses to trunk rotations under a stable head were driven by the stimulation velocity and acceleration and were highly direction sensitive. The interaction between vestibular and neck proprioceptive signals evoked facilitated or cancelled responses, allowing for multimodal coding of the body-in-space. Such coding, at the level of neural populations within the PIVC, may contribute to the distinction between active and passive motion, as previously reported in the vestibular nuclei and thalamus (Marlinski and McCrea, 2008b; McCrea et al., 1999). We also note that no response to static tilts of the animals was found in the PIVC by Grüsser and colleagues (Grüsser et al., 1982, 1990a,b), although recent electrophysiological studies indicated that PIVC neurons responded to both rotational and translational vestibular stimulation (Chen et al., 2010; Liu et al., 2010).

Human data. Functional neuroimaging studies performed in healthy subjects during caloric and galvanic vestibular stimulation have unanimously demonstrated activation of the posterior insula and temporo-parietal junction (TPJ), that could represent the human homologue of the monkey PIVC (Bense et al., 2001; Bottini et al., 1994, 1995, 2001; Bucher et al.,
1998; Dieterich et al., 2003; Eickhoff et al., 2006b; Emri et al., 2003; Fasold et al., 2002; Fink et al., 2003; Friberg et al., 1985; Hegemann et al., 2003; Indovina et al., 2005; Lobel et al., 1998; Marcelli et al., 2009; Miyamoto et al., 2007; Naito et al., 2003; Petit and Beauchamp, 2003; Schlindwein et al., 2008; Stephan et al., 2005; Suzuki et al., 2001; Tuohimaa et al., 1983; Vitte et al., 1996). These activations centered on the superior temporal gyrus, the posterior and anterior insula, or the inferior parietal lobule (Fig. 2B). However, we note that these different studies have relied on a large variety of neuroimaging methods and used various techniques to stimulate the vestibular apparatus (galvanic and caloric stimulation, auditory clicks), making it at present difficult to conclude whether these anatomical differences relate to differences in the technique and/or the procedures used. The exact location of human PIVC therefore remains to be determined (see also the clinical Section 4.1 below).

Finally, several neuroimaging studies have shown the implication of the anterior insula in vestibular processing, with activation extending sometimes into the adjacent inferior frontal gyrus (Bense et al., 2001; Bottini et al., 2001; Dieterich et al., 2003; Eickhoff et al., 2006b; Fasold et al., 2002; Stephan et al., 2005). The anterior insula has been proposed as a main region for interoception and bodily awareness (Craig, 2009) and vestibular signals could thus interact with this region to influence various aspects of spatial and bodily perception (Trousselard et al., 2004).

\subsubsection{Vestibular projections to the frontal cortex}

Animal data. Studies in animals showed vestibular projections to regions in the primary motor cortex (area 4) and premotor cortex (area 6) that are associated with motor and oculomotor control (Boisacq-Schepens and Hanus, 1972; Ebata et al., 2004; Fukushima et al., 2006, 2000, 2010; Fukushima and Kaneko, 1995; Sugiuchi et al., 2005). Ebata et al. (2004) showed
A

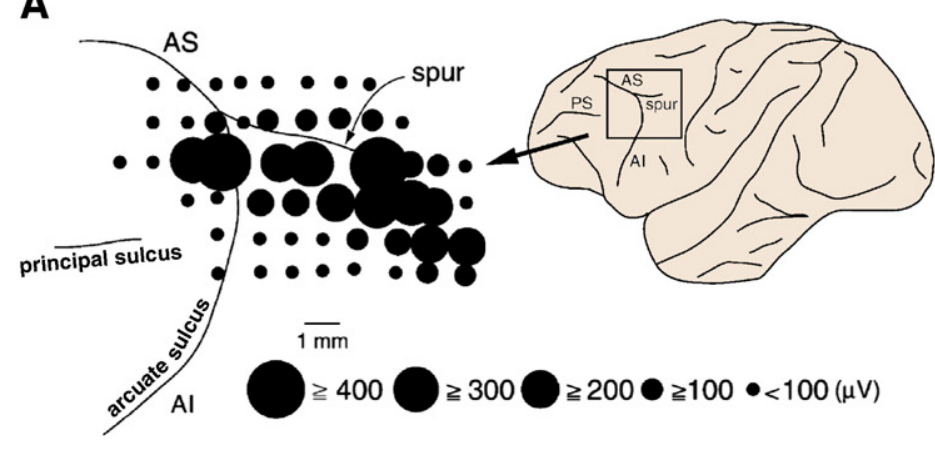

B

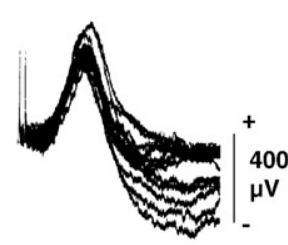

$10 \mathrm{~ms}$

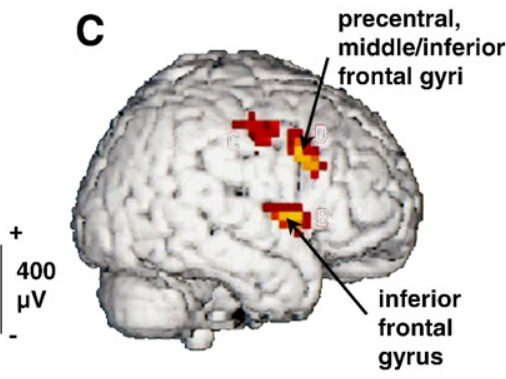

gyrus

Fig. 6 - Vestibular responses in the monkey and human frontal cortex. (A) Neurons located in the periacutate cortex of Japanese monkeys (Macaca fuscata) respond to electrical stimulation of the vestibular nerve. The size of the circles is proportional to the amplitude of the vestibular evoked-potential measured during electrical stimulation of the contralateral vestibular nerve. Inferior (AI) and superior (AS) ramus of the arcuate sulcus; PS: principal sulcus. Adapted from Ebata et al. (2004). (B) Evoked potentials found during electrical stimulation of the contralateral vestibular nerve, showing an early positive evoked-potential followed by a sustained, low amplitude, negative evoked-potential. Reproduced from Ebata et al. (2004). (C) Galvanic vestibular stimulation activated a region in the inferior frontal sulcus, overlapping with the precentral gyrus and the inferior and middle frontal gyri. Vestibular stimulation also activated the most inferior and posterior part of the right inferior frontal gyrus (pars opercularis) extending to the frontal operculum. Modified after Lobel et al. (1998). 
that potentials evoked by electrical stimulation of the vestibular nerve were localized in the periacutate cortex, mostly in the postarcuate cortex located around the spur of the arcuate sulcus, and to a lesser extent in area 6, medially to the superior ramus of the arcuate sulcus (Fig. 6A). Responses were evoked by electrical stimulation of both the ipsilateral and contralateral vestibular nerves (Fig. 6B). Studies in monkeys (Ebata et al., 2004; Fukushima et al., 2000, 2010) established that vestibular projections to areas around the arcuate sulcus and to the dorso-medial frontal cortex coincide with the frontal eye fields (FEF) and the supplementary eye fields in relation to the generation of saccades and smoothpursuit eye movements (Bruce et al., 1985; Schlag and SchlagRey, 1987). Activity in these regions might be partly related to the vestibulo-ocular reflex, although there is evidence that it is maintained during vestibulo-ocular reflex suppression and during smooth pursuit eye movements. Fukushima et al. (2000) proposed that populations of neurons in the FEF and supplementary eye fields are involved in pursuit-related vestibular functions and driven by both retinal image- and gaze-velocity signals.

Human data. In humans, galvanic and caloric vestibular stimulation were also found to activate several frontal regions including the primary motor cortex (precentral gyrus), the premotor cortex, as well as the superior (BA 10/8), middle (BA 9/8/6) and inferior (areas 44 and 47) frontal gyri (Bense et al., 2001; Emri et al., 2003; Fasold et al., 2002; Lobel et al., 1998; Miyamoto et al., 2007). During galvanic vestibular stimulation, Lobel et al. (1998) reported activation in the pars opercularis of the inferior frontal gyrus, and more dorsally in the inferior and medial frontal gyri (Fig. 6C). These authors argued that this activation is distinct from the FEF (that are located more anteriorly), and may represent the human homologue of the monkey's vestibular region described in the anterior ventral part of the premotor area 6. We note that activations in the lower part of the inferior frontal gyrus (pars opercularis), overlapping with Broca's area (sometimes extending to the anterior insula; Dieterich et al., 2003), have not been described as vestibular cortex in animals. On the contrary, other authors found frontal activations more dorsally in the "dorsolateral prefrontal cortex" (Fasold et al., 2002), or in the middle and superior frontal gyri (Bense et al., 2001; Dieterich et al., 2003; Miyamoto et al., 2007; Stephan et al., 2005), that have been proposed to represent vestibular processing in the human FEF in relation to the control of saccades, smooth-pursuit eye movements and nystagmus (Blanke et al., 2000b; Blanke and Seeck, 2003; Paus, 1996; Petit et al., 1997). These potential homologies between oculomotor and vestibular function in human and non-human primates should be regarded with caution as there may be subtle interspecies differences in anatomico-functional organization between area 6 and 8 (Tehovnik et al., 2000).

\subsubsection{Vestibular projections to the cingulate cortex}

Animal data. In the mesial frontal cortex, a "vestibular cingulate region" covering the cingulate sulcus of the monkey has been described (Guldin et al., 1992; Guldin and Grüsser, 1998). Although this region does not directly receive vestibular inputs, tracer studies showed that the vestibular cingulate region is densely connected with PIVC, area $3 a$ and VPS (see Fig. 5). Anatomical and behavioral observations suggest that the cingulate cortex takes part of a network involved in spatial coding and integration of visual and self-motion cues. In particular, this region contains head-direction cells and might be involved in path integration during navigation (Cooper et al., 2001; Taube, 1998).

Human data. Several neuroimaging studies in humans have also pointed to vestibular-related activations in the anterior (areas 24 and 32) and posterior cingulate cortex that could represent the homologue of the monkey vestibular cingulate region (Bense et al., 2001; Bottini et al., 1994; Fasold et al., 2002; Miyamoto et al., 2007; Suzuki et al., 2001).

\subsubsection{Vestibular projections to striate and extrastriate visual cortex}

Animal data. Animal studies have indicated vestibular projections to the cat's primary and secondary visual cortex (Grüsser et al., 1959; Grüsser and Grüsser-Cornehls, 1972; Vanni-Mercier and Magnin, 1982). According to Vanni-Mercier and Magnin (1982) vestibular stimulation modulates activity of $14 \%$ and $11 \%$ of neurons in area 17 and area 18, respectively, and of $21 \%$ of neurons in area 21 . Only $4 \%$ of the neurons were reported to be driven by vestibular stimulation in area 19. Vestibular projections have also been described in the monkey MST, a major brain region for visual motion perception as well as self-motion perception based on optic-flow (Bremmer et al., 1999; Duffy, 1998; Page and Duffy, 2003; Thier and Erickson, 1992).

Human data. Relatively few human studies have revealed occipital lobe contribution to vestibular processing. The lateral occipital gyrus has been found activated during caloric stimulation and this activation has been attributed to the MT/MST complex (Fasold et al., 2002). Bense et al. (2001) have proposed that the activation of the middle temporal gyrus (area 37) during galvanic vestibular stimulation may be the human homologue of the MT/MST complex of monkeys. In addition to these activations, several visual regions have been reported to be "deactivated" during vestibular stimulation (see Table 1). Brandt and colleagues showed that caloric vestibular stimulation reduced the blood flow in the posterior cerebral artery irrigating the visual cortex (Tiecks et al., 1996; Wenzel et al., 1996). Deactivation of the visual cortex may also concern the fusiform and inferior occipital gyri, together with a deactivation of the precuneus (Bense et al., 2001; Stephan et al., 2005) and prefrontal cortex (Lobel et al., 1998). On the basis of these data, reciprocal inhibitory visual-vestibular interactions have been hypothesized (Brandt et al., 1998). Finally, on a methodological point of view, we note that, even though most neuroimaging studies have been conducted with participants placed in darkness and with eyes closed, one can wonder what was the influence of the retinal image slip induced by caloric or galvanic stimulation in studies that used free vision. Indeed, the velocity of the caloric nystagmus was found to correlate with the brain activity in several regions overlapping with the vestibular cortex, including the insula, inferior parietal lobule, fusiform and lingual gyrus, and the cerebellum (Naito et al., 2003). These authors also demonstrated that visual fixation was related to a deactivation of the vestibular cortex.

\subsubsection{Vestibular projections to the hippocampus}

Animal data. Electrophysiological recordings in monkeys and rats showed that hippocampal activity is modulated by 
vestibular stimulation (Horii et al., 2004; O’Mara et al., 1994). First, the activity of hippocampal "place cells" - a category of pyramidal neurons from CA1 and CA3 hippocampal regions coding the animal location within the environment - is strongly tuned to vestibular input (Wiener et al., 2002). Second, "head direction cells" in the hippocampus, whose firing rate is modulated by the direction an animal is facing, are also strongly depending on vestibular information (Taube, 1998). Yet, the pathways through which vestibular cues reach the hippocampus are not yet clearly established, the main potential candidate being a pathway via the thalamus, the posterior parietal cortex and the perirhinal and entorhinal cortices, although pathways through PIVC or cingulate cortex are also possible (see Smith, 1997).

Human data. Vitte et al. (1996) demonstrated a predominant ipsilateral activation of the hippocampus and of the subiculum during caloric vestibular stimulation. This was later confirmed using the same kind of stimulation by Suzuki et al. (2001), who showed an additional activation of the parahippocampal gyrus (area 36). These sparse data may nevertheless suggest that vestibulo-hippocampal interactions are also present in humans. Indeed, deficits in spatial memory due to bilateral vestibular loss have been associated with hippocampal atrophy (Brandt et al., 2005).

\subsection{Connections between vestibular cortical areas}

Animal data. Based on these numerous vestibular cortical areas, and on the importance of multisensory integration that is occurring within these areas, it is generally thought that there is no primary vestibular cortex, in the sense that there is a primary visual, auditory, or somatosensory cortex. Thus, no single brain region seems to contain neurons exclusively afferented by vestibular input. All vestibular cortical areas are multimodal brain regions. In this multimodal network, the PIVC has been proposed to play a central role (Guldin and Grüsser, 1998). Injections of retrograde tracers in the PIVC showed that this region was the only vestibular area connected to all other vestibular regions (Grüsser et al., 1994, 1992). Fig. 7 summarizes the main connections between vestibular areas in the monkey's brain. The PIVC was shown to receive inputs from the premotor cortex (areas 6 and 8), somatosensory cortex (3av, 3aH and area 2), posterior parietal cortex (area 7), area T3, granular insula Ig, and to a less extent from the anterior cingulate gyrus. Area 3av receives projections from areas $6,4,3 b, 1,2,7$, from the secondary somatosensory cortex, the PIVC, the Ig, and the anterior cingulate regions (area 24 and the anterior cingulate sulcus).

Human data. Following observations in animals, some authors have described the PIVC as the core region of the human vestibular cortex (Brandt and Dieterich, 1999). We note that it is difficult to conclude about the presence of a primary vestibular cortex, or a core vestibular region in humans, based on existing PET and fMRI studies. Indeed, the poor temporal resolution of PET and AMRI makes it difficult to determine the time course of subcortical and cortical vestibular processing (but see (Marcelli et al., 2009)). Thus, none of these techniques has so far described a sequence of activation across cortical areas, or a simultaneous activation of cortical areas, following a vestibular stimulation. However, in an electroencephalography study in patients who underwent a curative vestibular neurectomy, electrical stimulation of the vestibular nerve was found to activate simultaneously five cortical regions within $6 \mathrm{~ms}$ (de Waele et al., 2001). According to these authors, a $6 \mathrm{~ms}$ latency is compatible with a trisynaptic pathway transmitting information from the vestibular nerve to the vestibular nuclei ( $2 \mathrm{~ms})$, from the vestibular nuclei to the thalamus $(1.5 \mathrm{~ms})$ and from the thalamus to the cerebral cortex (2.5 ms) (de Waele et al., 2001). This study confirmed the view according to which vestibular cues are transmitted to vestibular cortical areas through parallel pathways instead of reaching a primary vestibular cortex (that would have been activated earlier), and then being distributed to secondary areas (that would have been activated later). More work, especially using electrical neuroimaging methods and physiological vestibular stimulation, may be necessary to further investigate the location and timing of activation in the human vestibular cortex.
Java monkeys
Macaca fascicularis

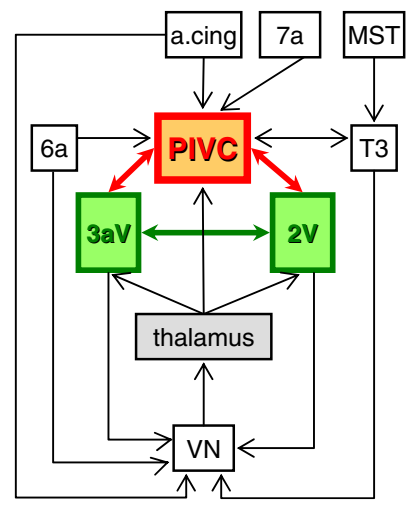

Squirrel monkeys

Saimiri sciureus

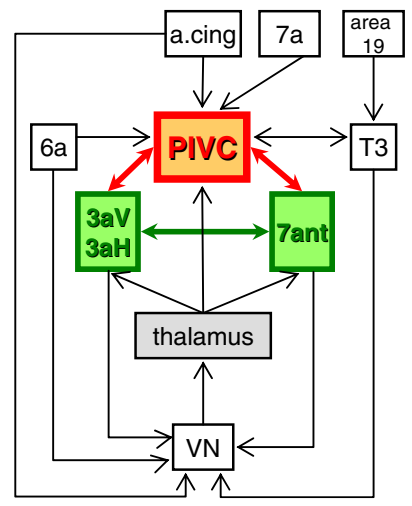

Marmoset monkeys

Callithrix jacchus

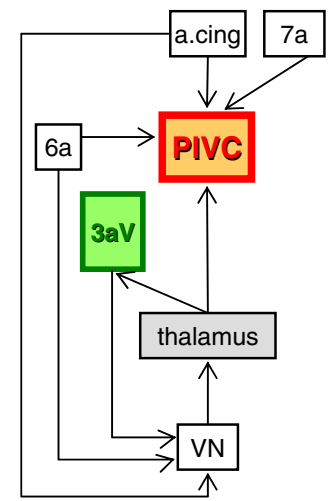

Fig. 7 - Cortico-cortical connections in the monkey vestibular cortex. Schematic representation of the connections between vestibular cortical areas showing similarities between Old World monkeys (Java monkeys, Macaca fascicularis) and New World monkeys (squirrel monkeys, Saimiri sciureus and marmoset monkeys, Callithrix jacchus). a.cing.: anterior cingulate region. Redrawn after Grüsser et al. (1994). 


\subsection{Hemispheric dominance of cortical vestibular projections}

Animal data. The laterality of the vestibular projections to the cortex has been investigated by electrophysiological recordings in animals using two different approaches. During electrical stimulation of the vestibular nerve, vestibular-evoked potentials were recorded in different cortical regions and the latency and amplitude of the potentials were compared in the ipsilateral and contralateral cortex. The type of response to rotations of the animal was also investigated, indicating if most of the neurons responded to ipsilateral or contralateral rotations. However, because vestibular signals have been transmitted through several synapses before reaching the cortex, and because during animal rotations both labyrinths are stimulated (activated or inhibited), the data are more difficult to interpret. The use of different research protocols for stimulating the vestibular receptors or the vestibular nerve and for recording the responses in the cortex may have further influenced the reported laterality of cortical responses in the different studies in animals.

In the primary somatosensory cortex Fredrickson et al. (1966) showed bilateral vestibular evoked-potentials in area $2 \mathrm{v}$ during electrical stimulation of the vestibular nerve. However, they noted that responses were almost exclusively contralateral, although the onset of responses was recorded in the ipsilateral and contralateral somatosensory cortex with similar latencies. Vestibular-evoked potentials were found with a latency of 5 $-6 \mathrm{~ms}$, which was consistent with the findings in humans (de Waele et al., 2001). Moreover, the same area was found to contain approximately the same proportion of neurons activated by natural vestibular stimulation directed to the ipsilateral side $(50 \%$ of neurons in area $2 \mathrm{v}$ were type I neurons, i.e. activated during yaw rotations to the side ipsilateral to the recording site) and to the contralateral side ( $48 \%$ were type II neurons, i.e. activated during yaw rotations to the side contralateral to the recording site) (Büttner and Buettner, 1978). This indicates that neurons in the somatosensory cortex were equally activated by vestibular afferents originating from the ipsilateral and contralateral semicircular canals. The patterns of laterality need to be interpreted with caution because of several synapses involved in transmitting the information from the labyrinth to the cortex and because of bilateral vestibular projections to the thalamus and the cortex. Similarly to area $2 \mathrm{v}$, the vestibular evokedpotentials recorded in area $3 \mathrm{aV}$ during electrical stimulation of the vestibular nerve were bilateral, but the amplitude of the evoked-potentials in area $3 \mathrm{aV}$ was much larger in the contralateral side of the stimulation (Ödkvist et al., 1974). Consistent observations have been made in the frontal periarcuate cortex, where vestibular evoked-potentials were recorded after electrical stimulation of both the contralateral and ipsilateral labyrinths (Ebata et al., 2004). These authors reported that the contralateral vestibular evoked-potentials had usually a larger amplitude than the ipsilateral evoked-potentials. In contrast with these studies, we note that Kempinsky (1951) reported in an early study in the cat that ipsilateral and contralateral vestibular projections to the somatosensory cortex were "symmetrical in extent and location."

The electrophysiological studies by Grüsser and colleagues in Java monkeys have provided detailed description of the responses of PIVC neurons to natural vestibular stimulation (rotations) (Grüsser et al., 1982, 1990a,b, 1994). These authors showed that, as classically observed in the vestibular nuclei and thalamic nuclei, PIVC comprises type I neurons (activated during yaw and roll rotations to the side ipsilateral to the recording site, and during nose-up rotations in the pitch plane), type II neurons (activated during yaw and roll rotations to the side contralateral to the recording site, and during nosedown rotations in the pitch plane) and type III neurons (activated for both directions of rotation) [according to the classification by Duensing and Schaefer, 1958]. Whatever the plane of rotation, most of the PIVC neurons display type II responses. More recently, Liu et al. (2010) confirmed that most PIVC neurons ( 59\%) had a type II response during yaw rotations. Similar directional sensitivity to yaw rotations (with a majority of type II responses) has been reported in the vestibular nuclei (Buettner et al., 1978) as well as in the vestibular thalamus (Büttner et al., 1977; Marlinski and McCrea, 2008a). Based on these observations, Grüsser et al. (1990b) proposed that "the distribution of directional sensitivity is not essentially modified during signal transmission from the vestibular nuclei to the 'vestibular cortex'."

Human data. Several neuroimaging studies have revealed that vestibular projections are bilateral, with activations found on both cerebral hemispheres, for example during unilateral caloric vestibular stimulation (Bottini et al., 1994; Dieterich et al., 2003). Bilateral activations have also been reported when using unilateral galvanic vestibular stimulation, together with transcranial Doppler ultrasound, showing a significant blood flow increase in both cerebral hemispheres (Schlosser et al., 2008). In addition to this bilateral pattern of vestibular projections, a hemispheric dominance was revealed depending on both the stimulated ear and the participant's handedness.

In a series of PET and FMRI studies conducted in healthy volunteers and neurological patients, Dieterich and colleagues investigated whether the side of caloric vestibular stimulation as well as manual dominance (or handedness) influence the activation of the vestibular cortex (Dieterich et al., 2003, 2005a, 2005b; Janzen et al., 2008). The authors observed that both sides of peripheral stimulation as well as handedness of the subjects modulated activation of vestibular cortex. First, they described a predominant activation of ipsilateral vestibular cortex following right or left caloric ear irrigation (with warm water at $44^{\circ} \mathrm{C}$ ). In addition, the authors observed that this pattern of cortical activation after unilateral caloric ear irrigation was additionally and strongly modulated by the handedness of the subjects. Thus, Dieterich et al. (2003) report that the right hemisphere in right-handers, and the left hemisphere in left-handers, showed strongest vestibular cortical activations, respectively, and that this was further modulated by which ear was stimulated. Based on the interaction with handedness, it was argued by the authors that the vestibular system and its cortical projections may influence the development of handedness in humans. While more work is needed on this topic, the finding of stronger vestibular responses in the hemisphere ipsilateral to the stimulated ear is consistent with electroencephalography recordings during electrical stimulation of the vestibular nerve in patients undergoing a unilateral vestibular neurectomy. De Waele et al. (2001) reported that early vestibular evoked-potentials recorded at electrodes located ipsilateral to 
the side of stimulation were larger than those recorded contralaterally. These patterns of vestibular projections in humans, suggesting an importance of the ipsilateral pathways, do thus not fully correspond with animal data, that have stressed bilateral pathways with a contralateral dominance. In addition, this pattern of hemispheric projection for the vestibular system thus seems to differ from the strongly lateralized contralateral pathways in the visual and somatosensory systems and the bilateral, but predominant contralateral pathways in the auditory system (Squire et al., 2008).

\section{Human vestibular cortex in neurological disease}

4.1. The search for human PIVC: electrical stimulation and brain damage to the insula and temporo-parietal junction

Several reports from earlier as well as more recent literature on focal brain stimulation in conscious epileptic patients and brain-damaged patients are compatible with the hypothesis that the posterior insula/TPJ represents the human homologue of the monkey PIVC (data summarized in Fig. 8A), although its exact location in the human brain is still debated (Eickhoff et al., 2006b; Kahane et al., 2003; Lobel et al., 1998). In patients operated on for focal epilepsy, Penfield (1957) reported that electrical cortical stimulation of the superior temporal gyrus evoked vestibular illusions described as "dizziness, swinging, spinning" (case \#94), "sinking feeling and sensation of full head" (case \#5), or "head rest seems to be jumping up and down" (case \#25). Congruently, epileptic patients with vestibular aurae have epileptic foci and brain lesions surrounding the superior temporal gyrus and the temporo-parietal cortex (Penfield and Kristiansen, 1951; Smith, 1960). This was confirmed more recently by Kahane et al. (2003) in a large population of epileptic patients. They showed that electrical stimulation applied at a widespread region of cortex - spanning different loci in the superior and middle temporal gyri - elicited illusion such as "levitation, lightness" (case \#9712b), "rolling forwards" (case \#9712c) and "head spinning" (case \#9701) (Fig. 8A). In addition to vestibular sensations, stimulation of the superior temporal gyrus has been shown to evoke oculomotor responses (Best et al., 2009). Based on these clinical and neuroimaging data (see Section 3.1.2), it is therefore not clear whether the human PIVC is located in the posterior insula, inferior parietal lobule, superior temporal gyrus, or at the junction between these regions. On the basis of studies in epileptic patients, Smith (1960) proposed that "the vestibulo-psychic area [...] may have its epicenter in the region of the angular gyrus" (Fig. 8A). Brandt and colleagues suggested that the posterior insula could represent the human PIVC (Brandt and Dieterich, 1999). In keeping with this view, there is evidence that lesions of the insula and superior/middle temporal gyrus can impair the perception of the visual vertical (Barra et al., 2010; Brandt et al., 1994; Hegemann et al., 2004) and induce rotational vertigo and unsteadiness (Boiten et al., 2003; Brandt et al., 1995; Cereda et al., 2002; Erbayat Altay et al., 2005; Nicita et al., 2010). Kahane and colleagues questioned whether a region they called temporo-peri-Sylvian vestibular cortex, "roughly distributed around the posterior part of the insula but clearly
A
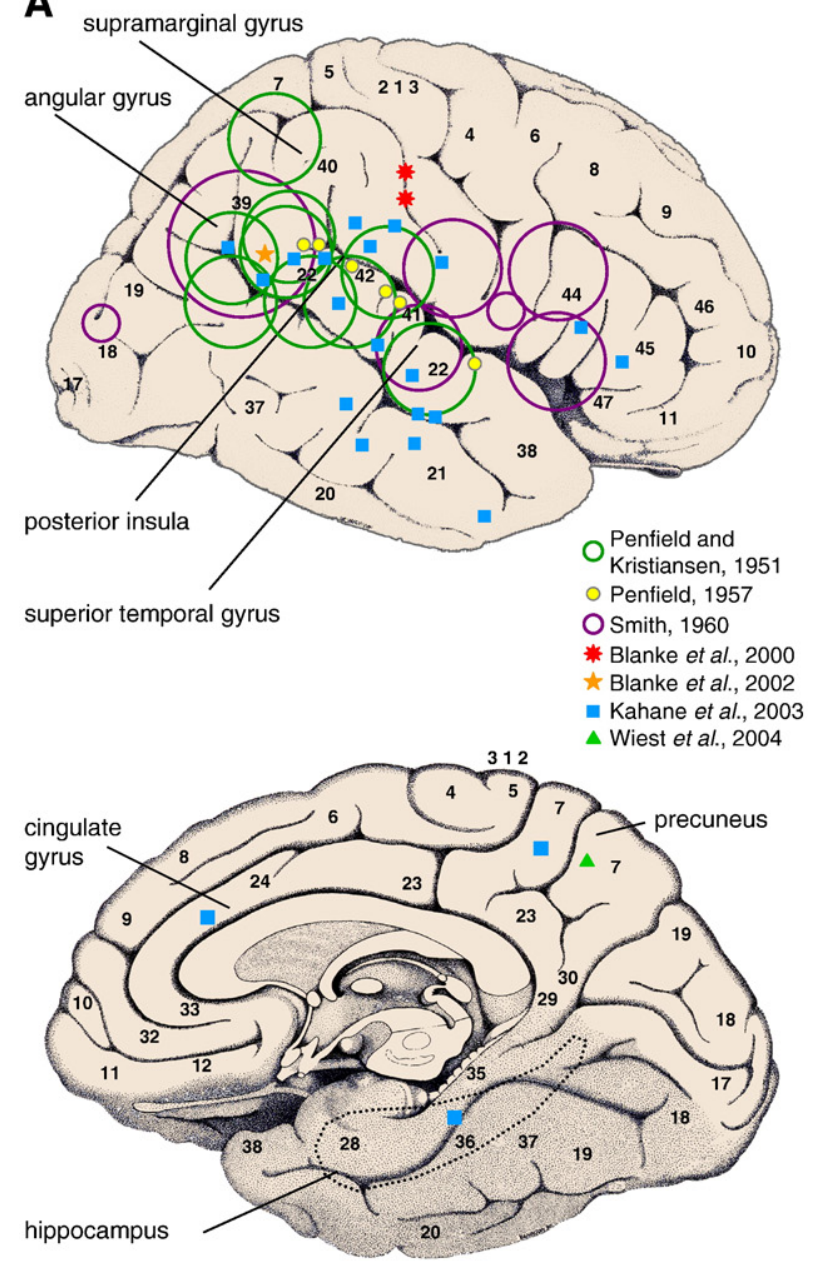

B

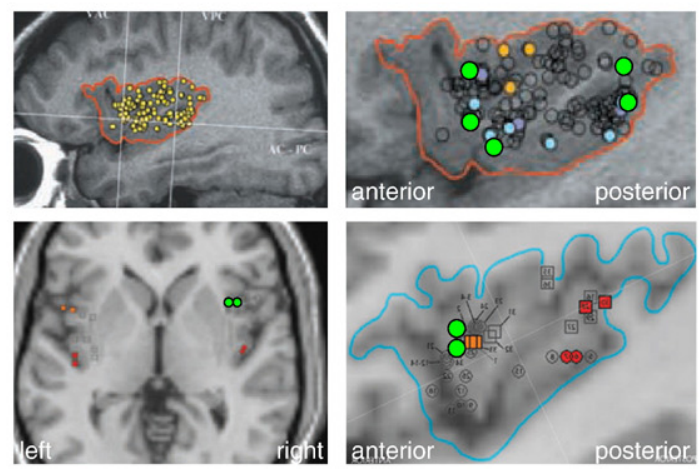

Fig. 8 - Vestibular areas described in epileptic patients. (A) Green and purple open circles represent the location of epileptogenic lesions responsible for vestibular sensations. Filled symbols represent the site at which focal electrical stimulation of the cortex evoked vestibular illusions in awake epileptic patients. The numbers on the cortex refer to the cytoarchitectonic areas defined by Brodmann (modified after Duvernoy, 1999). (B) Site of electrical stimulation of the insula evoking vestibular illusions (green dots). After Isnard et al. (2004) and Nguyen et al. (2009). 
located more superficially, without involving the insula strictly speaking" could be the human homologue of PIVC. The vestibular cortex would therefore be localized more superficially and would not necessarily involve the insula (Kahane et al., 2003; Lobel et al., 1998). These authors also based their conclusion on the finding that electrical stimulation of the insula only rarely evoked conscious vestibular sensations in epileptic patients (see Fig. 8B and Isnard et al., 2004; Nguyen et al., 2009; Ostrowsky et al., 2002; Penfield and Faulk, 1955; Selimbeyoglu and Parvizi, 2010). This latter view is in line with recent fMRI and cytoarchitectonic mapping of the human parietal operculum and posterior insula (Eickhoff et al., 2006a; Kurth et al., 2010), suggesting that the human analogue of the PIVC may be localized in the parietal operculum (see Fig. 5C: area $\mathrm{OP} 2$, at the junction of the posterior parietal operculum with the insular and retroinsular areas) rather than in the posterior insula (Eickhoff et al., 2006b). We finish this paragraph by noting that other clinical observations also suggested vestibular processing in the anterior insula as lesions confined to this region may induce vertigo and postural imbalance (Papathanasiou et al., 2006).

\subsection{Electrical stimulation and brain damage to the parietal cortex}

In the human vestibular cortex, area $2 \mathrm{v}$ is likely homologous to a parietal area described by Foerster (1936) during electrical cortical stimulation in awake patients undergoing brain surgery. Stimulation of a region deep within the intraparietal sulcus elicited sensations of full body rotations in space. This has been corroborated more recently by describing human area $2 \mathrm{v}$ posteriorly to somatosensory areas involved in lower face and tongue representation and thus somewhat more laterally than in animals (Blanke et al., 2000a) (Fig. 4C). The contribution of the anterior intraparietal sulcus to vestibular processing is also supported by recent neurological observation showing that a lesion of the right central sulcus extending to the border of the intraparietal sulcus might induce rotational vertigo (patient \#1 from (Urasaki and Yokota, 2006).

Electrical stimulation applied to parietal regions located more posteriorly, in area 39 (Blanke et al., 2002) and area 40 (Blanke et al., 2000a; Kahane et al., 2003), may also evoke conscious vestibular illusions in epileptic patients. Rotational vertigo may also result from lesion of the inferior parietal cortex or its adjacent parietal white matter (patient \#2 from Urasaki and Yokota, 2006; Erbayat Altay et al., 2005; Nicita et al., 2010; Schneider et al., 2006). Finally, human mesial parietal cortex may harbor a vestibular region because vestibular illusions were evoked in epileptic patients during electrical stimulation of the precuneus (Kahane et al., 2003; Wiest et al., 2004).

\subsection{Other vestibular cortical areas}

Although the influence of stimulation and damage to the insula and TPJ has been demonstrated in several clinical studies, the contribution of other cortices is rather sparse in the neurological literature. A contribution of the frontal cortex to vestibular processing is suggested by neurological observations reporting rotational vertigo due to epilepsy and brain damage in frontal cortex (Hochman, 1983; Kahane et al., 2003;
Kluge et al., 2000; Lopez et al., 2010a; Nicita et al., 2010). The implication of the cingulate cortex and of the hippocampus is also suggested by the effects of electrical stimulation in epileptic patients (Kahane et al., 2003), but this has to be confirmed in larger clinical populations.

\section{Corticofugal projections to the brainstem}

Vestibular cortical areas, that receive vestibular inputs from the vestibular nuclei via the thalamus, send in turn projections to the vestibular regions in the brainstem, including the vestibular nuclei, the parabrachial nuclei and the nucleus prepositus hypoglossi. Anatomical studies using retrograde tracer injections into the vestibular nuclei, or anterograde tracer injections into the vestibular cortical areas, have been conducted in different monkey species (Akbarian et al., 1993, 1994; FaugierGrimaud and Ventre, 1989; Guldin et al., 1993; Ventre and Faugier-Grimaud, 1988), in the cat (Wilson et al., 1999) and in the rat (Nishiike et al., 2000). These studies showed that vestibular nuclei receive direct monosynaptic projections from PIVC, areas $2 \mathrm{v}, 3 \mathrm{av}, 7$, VPS area (in area T3), premotor cortex (area 6), and anterior cingulate cortex (areas 23/6). As a general rule, all nuclei from the vestibular nuclear complex receive projections from both cerebral hemispheres and from most of these areas. However, the PIVC and surrounding areas project mainly to the ipsilateral vestibular nuclei, while the premotor cortex (area 6) and the somatosensory cortex (areas 3av and 2v) project preferentially to the contralateral vestibular nuclei (Akbarian et al., 1994). In addition, there are two subdivisions in the corticofugal pathways with respect to the functional specialization of the vestibular cortical areas and vestibular nuclei: PIVC, somatosensory area $2 \mathrm{v}$ and cingulate areas $23 / 6$ innervated preferentially the vestibular nuclei strongly connected to the oculomotor structures (MVN, SVN). The premotor cortex (area 6) and somatosensory area 3av innervated mainly the vestibular nuclei involved in vestibulo-spinal functions (LVN and MVN). Such distinct corticofugal projections may represent the substrate of the descending and specialized control of vestibulo-oculomotor and vestibulo-spinal functions during body motion.

The corticofugal influence on vestibular nuclei is further supported by electrophysiological recordings in vestibular nuclei while different vestibular cortical areas are electrically stimulated. Stimulation of areas 2 and 3a (Wilson et al., 1999), area 4 (Licata et al., 1987; Licata et al., 1990) and the FEF (Fukushima et al., 1984) modified the responses of vestibular nuclei neurons. Wilson et al. (1999) showed that these responses are complex and can manifest as facilitation or inhibition of the vestibular neurons response, or by a combination of these effects. In addition, on the basis of the latency and properties of the effects, they proposed that corticofugal control of the vestibular nuclei would be evoked by polysynaptic pathways. The neural mechanisms of the corticofugal influence on vestibulo-oculomotor and vestibulo-spinal commands remain to be clarified.

In addition to corticofugal projections to the vestibular nuclei, cortical projections to brainstem oculomotor and limbic structures have been described (Faugier-Grimaud and Ventre, 1989; Fries, 1984). For example, Faugier-Grimaud and Ventre 
(1989), showed projections from the posterior parietal cortex (area 7) to the nucleus propositus hypoglossi, the dorsolateral pontine nucleus, the accessory nucleus of Darkschewitsch, as well as the intermediate and deep layers of the superior colliculus. Projections to the superior colliculus may be involved in the control of saccades and smooth pursuit eye movements (Pare and Wurtz, 1997). In addition, projections from the insular cortex to the parabrachial nucleus have been reported in the rat (Allen et al., 1991; Saper, 1982). The parabrachial nucleus, that has been shown to respond to vestibular stimulation (McCandless and Balaban, 2010), is connected with the vestibular nuclei, the amygdala and the hypothalamus (Balaban, 2004). Based on this pattern of projections, it has been proposed that the parabrachial nucleus is involved in limbic and autonomic control of vestibular responses, and vice versa (Balaban, 1999).

Some clinical and behavioral arguments support the existence of cortico-vestibular interactions in animals and humans. Analysis of the vestibulo-ocular reflex after lesion of area 7 in monkeys showed a strong asymmetry of this reflex, with a decreased gain for rotations of the animal towards the side contralateral to the lesion and an increased gain for rotations to the ipsilesional side (Ventre and Faugier-Grimaud, 1986). Similar observations were done in patients with lesions involving the temporo-occipito-parietal junction, essentially in the right hemisphere (Ventre-Dominey et al., 2003). Finally, some behavioral studies suggested an influence of attentional and topdown mechanisms on vestibular functions, as revealed by the analysis of the vestibulo-ocular reflex (for reviews see Berthoz, 1996; Fukushima, 1997). For example, the simple imagination of a head-fixed target can suppress the vestibulo-ocular reflex in subjects passively rotated in darkness (Melvill Jones et al., 1984). In the same line, cognitive tasks (e.g. mental calculation) can influence postural stability (Woollacott and Shumway-Cook, 2002). Altogether, these data are suggestive of a role of the corticofugal projections in controlling vestibulo-oculomotor and vestibulo-spinal functions (Berthoz, 1996), but this contribution deserves further investigations in humans.

\section{Summary and future perspectives}

We have summarized data on many thalamic and cortical regions that are involved in vestibular processing. Bilateral projections from the vestibular nuclei send signals to the thalamus of rats, cats and primates. Anatomical and electrophysiological studies in animals identified vestibular neurons located in many thalamic nuclei including the VPL, VPI, VPM, VPP, VA - VL, IL, MGN, LGN, pulvinar, SGN and LD. These thalamic neurons are characterized by very similar responses that have been described for vestibular nuclei neurons. Notably, the role of different populations of thalamic primate neurons in distinguishing translations us. head tilts with respect to gravity has been described. Thalamic vestibular populations also distinguished between active and passive head movements, showing an integration with motor signals at the thalamic level. However, the exact role of these thalamic nuclei in relaying and modulating vestibular signals remains to be investigated in a comparable fashion as done for other major sensory systems
(Sherman and Guillery, 2002). Compared to these sparse but detailed findings on animal vestibular thalamus, understanding of vestibular functions in the human thalamus has suffered from the difficulty to delineate activations in the human thalamus. Thus, anatomical studies need to be conducted combining ultrahigh field neuroimaging and other MR-techniques such as diffusion tractography-based segmentation of the thalamus. This will help to better understand thalamic functions and structure in humans and to describe pathways from the vestibular thalamus to the cerebral cortex as previously done for other sensory modalities (Behrens et al., 2003; Johansen-Berg et al., 2005).

Concerning vestibular cortex, there is no unique and welldefined primary vestibular cortex (koniocortex) comparable to primary sensory cortex for vision, somatosensation, and audition. It has been proposed that the PIVC is the core vestibular region in non-human primates (Guldin and Grüsser, 1998). Other vestibular regions include somatosensory cortex (area 2v, 3av), intraparietal sulcus (area VIP and MIP), posterior parietal cortex (area 7), area MST, frontal cortex (FEF), cingulum (cingulate vestibular cortex) and hippocampus. These regions are involved in various functions ranging from the perception of body rotation and translation, oculomotor and balance control, visual and postural vertical perception, to spatial navigation and memory (Angelaki et al., 2009; Berthoz, 1996; Smith et al., 2010).

In humans, the growing amount of neuroimaging data over the last two decades has allowed to also describe a highly distributed vestibular cortical network. The vestibular regions described in the human cortex closely match those found in animals: they were found in the PIVC (posterior insula), superior temporal gyrus, inferior parietal lobule (angular and supramarginal gyrus), somatosensory cortex, precuneus, cingulate gyrus, frontal cortex (motor cortex and FEF) and hippocampus. With respect to the localization of the core vestibular region in humans, further studies need to be conducted to determine more precisely whether the human PIVC region is located deep in the lateral sulcus in the posterior insula (Brandt and Dieterich, 1999), more superficially in the temporo-peri-Sylvian vestibular cortex (Kahane et al., 2003), or more dorsally in the parietal operculum (Eickhoff et al., 2006b). Combining neuroimaging in healthy participants using electroencephalography (with its high temporal resolution) and MRI-based techniques (with their high spatial resolution), jointly with vestibular stimulation, may further advance on the anatomy of the vestibular cortex. This should also include the recording of local field potentials in epileptic patients while undergoing invasive medical explorations (Engel et al., 2005; Jerbi et al., 2010). A further limitation we note is that most neuroimaging studies in humans relied on artificial vestibular stimulation, such as caloric or galvanic vestibular stimulation, that also activate somatosensory, thermic, nociceptive and gustatory pathways, in addition to the vestibular system. Physiological vestibular stimulation, allowing to distinguish otolith from semicircular canal afferents, potentially in combination with scalp electroencephalography recordings, may also be relevant to achieve further advances in understanding the vestibular system in humans (see, e.g., Baudonniere et al., 1999; Hood and Kayan, 1985). Finally, these data should be compared with the effects of cortical stimulation (Blanke et al., 2000b; Kahane et al., 2003; Selimbeyoglu and 
Parvizi, 2010) and focal brain damage on vestibular sensation and function in neurological patients in order to precisely delineate the extent of PIVC and the other regions constituting the human vestibular cortex.

The description of the extent of vestibular projections to various multisensory cortical regions involved in spatial and bodily representation has also helped understanding the large range of spatial and bodily deficits observed in patients with vestibular dysfunctions (Borel et al., 2008; Smith et al., 2005). The description of the anatomy and function of the vestibular cortex is thus not only necessary for systems neuroscience, but should also further our understanding of clinical aspects of vestibular disorders and the role of the vestibular cortical system in perception, cognition, and consciousness. The vestibular system has been involved in a variety of perceptive and cognitive functions based on behavioral effects reported after caloric or galvanic vestibular stimulation in healthy humans (Lenggenhager et al., 2008; Lopez and Blanke, 2007; Lopez et al., 2010b; Miller and Ngo, 2007), and such stimulation may have beneficial effects on deficits regarding external and corporeal space in brain-damaged patients (Bottini et al., 1995; McGeoch et al., 2008; Vallar et al., 1993).

\section{Acknowledgments}

The authors are supported by the Swiss National Science Foundation (SINERGIA CRSII1-125135/1).

\section{REFEREN C ES}

Akbarian, S., Grüsser, O.J., Guldin, W.O., 1992. Thalamic connections of the vestibular cortical fields in the squirrel monkey (Saimiri sciureus). J. Comp. Neurol. 326, 423-441.

Akbarian, S., Grüsser, O.J., Guldin, W.O., 1993. Corticofugal projections to the vestibular nuclei in squirrel monkeys: further evidence of multiple cortical vestibular fields. J. Comp. Neurol. 332, 89-104

Akbarian, S., Grüsser, O.J., Guldin, W.O., 1994. Corticofugal connections between the cerebral cortex and brainstem vestibular nuclei in the macaque monkey. J. Comp. Neurol. 339, 421-437.

Aldes, L.D., 1988. Thalamic connectivity of rat somatic motor cortex. Brain Res. Bull. 20, 333-348.

Alitto, H.J., Usrey, W.M., 2003. Corticothalamic feedback and sensory processing. Curr. Opin. Neurobiol. 13, 440-445.

Allen, G.V., Saper, C.B., Hurley, K.M., Cechetto, D.F., 1991. Organization of visceral and limbic connections in the insular cortex of the rat. J. Comp. Neurol. 311, 1-16.

Angelaki, D.E., Cullen, K.E., 2008. Vestibular system: the many facets of a multimodal sense. Annu. Rev. Neurosci. 31, 125-150.

Angelaki, D.E., Shaikh, A.G., Green, A.M., Dickman, J.D., 2004. Neurons compute internal models of the physical laws of motion. Nature 430, 560-564.

Angelaki, D.E., Klier, E.M., Snyder, L.H., 2009. A vestibular sensation: probabilistic approaches to spatial perception. Neuron 64, 448-461.

Augustine, J.R., 1996. Circuitry and functional aspects of the insular lobe in primates including humans. Brain Res. Brain Res. Rev. 22, 229-244.

Balaban, C.D., 1999. Vestibular autonomic regulation (including motion sickness and the mechanism of vomiting). Curr. Opin. Neurol. 12, 29-33.
Balaban, C.D., 2004. Projections from the parabrachial nucleus to the vestibular nuclei: potential substrates for autonomic and limbic influences on vestibular responses. Brain Res. 996, 126-137.

Barmack, N.H., 2003. Central vestibular system: vestibular nuclei and posterior cerebellum. Brain Res. Bull. 60, 511-541.

Barra, J., Marquer, A., Joassin, R., Reymond, C., Metge, L., Chauvineau, V., Perennou, D., 2010. Humans use internal models to construct and update a sense of verticality. Brain 133, 3552-3563.

Baudonniere, P.M., Belkhenchir, S., Lepecq, J.C., Mertz, S., 1999. Otolith-vestibular-evoked potentials in humans. Intensity, direction of acceleration (Z+, Z-), and BESA modeling of generators. Ann. NY Acad. Sci. 871, 384-386.

Behrens, T.E., Johansen-Berg, H., Woolrich, M.W., Smith, S.M., Wheeler-Kingshott, C.A., Boulby, P.A., Barker, G.J., Sillery, E.L., Sheehan, K., Ciccarelli, O., Thompson, A.J., Brady, J.M., Matthews, P.M., 2003. Non-invasive mapping of connections between human thalamus and cortex using diffusion imaging. Nat. Neurosci. 6, 750-757.

Bense, S., Stephan, T., Yousry, T.A., Brandt, T., Dieterich, M., 2001. Multisensory cortical signal increases and decreases during vestibular galvanic stimulation (fMRI). J. Neurophysiol. 85, 886-899.

Berthoz, A., 1996. How does the cerebral cortex process and utilize vestibular signals? In: Baloh, R.W., Halmagyi, G.M. (Eds.), Disorders of the Vestibular System. Oxford University Press, New York, pp. 113-125.

Berthoz, A., 2000. The Brain's Sense of Movement, Vol. Harvard University Press, Cambridge, MA.

Berthoz, A., Vidal, P.P., 1993. Noyaux vestibulaires et vertiges, Vol. Arnette, Paris.

Berthoz, A., Israel, I., Georges-Francois, P., Grasso, R., Tsuzuku, T., 1995. Spatial memory of body linear displacement: what is being stored? Science 269, 95-98.

Best, C., Stefan, H., Hopfengaertner, R., Dieterich, M., 2009. Effects of electrical stimulation in vestibular cortex areas in humans. J. Neurol. Sci.

Blanke, O., Seeck, M., 2003. Direction of saccadic and smooth eye movements induced by electrical stimulation of the human frontal eye field: effect of orbital position. Exp. Brain Res. 150, 174-183.

Blanke, O., Perrig, S., Thut, G., Landis, T., Seeck, M., 2000a. Simple and complex vestibular responses induced by electrical cortical stimulation of the parietal cortex in humans. J. Neurol. Neurosurg. Psychiatry 69, 553-556.

Blanke, O., Spinelli, L., Thut, G., Michel, C.M., Perrig, S., Landis, T., Seeck, M., 2000b. Location of the human frontal eye field as defined by electrical cortical stimulation: anatomical, functional and electrophysiological characteristics. NeuroReport 11, 1907-1913.

Blanke, O., Ortigue, S., Landis, T., Seeck, M., 2002. Stimulating illusory own-body perceptions. Nature 419, 269-270.

Blum, P.S., Gilman, S., 1979. Vestibular, somatosensory, and auditory input to the thalamus of the cat. Exp. Neurol. 65, 343-354

Blum, P.S., Abraham, L.D., Gilman, S., 1979a. Vestibular, auditory, and somatic input to the posterior thalamus of the cat. Exp. Brain Res. 34, 1-9.

Blum, P.S., Day, M.J., Carpenter, M.B., Gilman, S., 1979b. Thalamic components of the ascending vestibular system. Exp. Neurol. 64, 587-603.

Boisacq-Schepens, N., Hanus, M., 1972. Motor cortex vestibular responses in the chloralosed cat. Exp. Brain Res. 14, 539-549.

Boiten, J., Wilmink, J., Kingma, H., 2003. Acute rotatory vertigo caused by a small haemorrhage of the vestibular cortex. J. Neurol. Neurosurg. Psychiatry 74, 388.

Borel, L., Lopez, C., Péruch, P., Lacour, M., 2008. Vestibular syndrome: a change in internal spatial representation Neurophysiol. Clin. 38, 375-389. 
Bottini, G., Sterzi, R., Paulesu, E., Vallar, G., Cappa, S.F., Erminio, F., Passingham, R.E., Frith, C.D., Frackowiak, R.S., 1994. Identification of the central vestibular projections in man: a positron emission tomography activation study. Exp. Brain Res. 99, 164-169.

Bottini, G., Paulesu, E., Sterzi, R., Warburton, E., Wise, R.J., Vallar, G., Frackowiak, R.S., Frith, C.D., 1995. Modulation of conscious experience by peripheral sensory stimuli. Nature 376, 778-781.

Bottini, G., Karnath, H.O., Vallar, G., Sterzi, R., Frith, C.D., Frackowiak, R.S., Paulesu, E., 2001. Cerebral representations for egocentric space: functional-anatomical evidence from caloric vestibular stimulation and neck vibration. Brain 124, 1182-1196.

Brandt, T., Dieterich, M., 1994. Vestibular syndromes in the roll plane: topographic diagnosis from brainstem to cortex. Ann. Neurol. 36, 337-347.

Brandt, T., Dieterich, M., 1999. The vestibular cortex. Its locations, functions, and disorders. Ann. NY Acad. Sci. 871, 293-312.

Brandt, T., Dieterich, M., Danek, A., 1994. Vestibular cortex lesions affect the perception of verticality. Ann. Neurol. 35, 403-412.

Brandt, T., Botzel, K., Yousry, T., Dieterich, M., Schulze, S., 1995. Rotational vertigo in embolic stroke of the vestibular and auditory cortices. Neurology 45, 42-44.

Brandt, T., Bartenstein, P., Janek, A., Dieterich, M., 1998. Reciprocal inhibitory visual-vestibular interaction. Visual motion stimulation deactivates the parieto-insular vestibular cortex. Brain 121, 1749-1758.

Brandt, T., Schautzer, F., Hamilton, D.A., Bruning, R., Markowitsch, H.J., Kalla, R., Darlington, C., Smith, P., Strupp, M., 2005. Vestibular loss causes hippocampal atrophy and impaired spatial memory in humans. Brain 128, 2732-2741.

Bremmer, F., Kubischik, M., Pekel, M., Lappe, M., Hoffmann, K.P., 1999. Linear vestibular self-motion signals in monkey medial superior temporal area. Ann. NY Acad. Sci. 871, 272-281.

Bremmer, F., Schlack, A., Duhamel, J.R., Graf, W., Fink, G.R., 2001. Space coding in primate posterior parietal cortex. Neuroimage 14, S46-S51.

Bremmer, F., Klam, F., Duhamel, J.R., Ben Hamed, S., Graf, W., 2002. Visual-vestibular interactive responses in the macaque ventral intraparietal area (VIP). Eur. J. Neurosci. 16, 1569-1586.

Bruce, C.J., Goldberg, M.E., Bushnell, M.C., Stanton, G.B., 1985. Primate frontal eye fields II. Physiological and anatomical correlates of electrically evoked eye movements. J. Neurophysiol. 54, 714-734.

Bucher, S.F., Dieterich, M., Seelos, K.C., Brandt, T., 1997. Sensorimotor cerebral activation during optokinetic nystagmus. A functional MRI study. Neurology 49, 1370-1377.

Bucher, S.F., Dieterich, M., Wiesmann, M., Weiss, A., Zink, R., Yousry, T.A., Brandt, T., 1998. Cerebral functional magnetic resonance imaging of vestibular, auditory, and nociceptive areas during galvanic stimulation. Ann. Neurol. 44, 120-125.

Buettner, U.W., Buttner, U., Henn, V., 1978. Transfer characteristics of neurons in vestibular nuclei of the alert monkey. J. Neurophysiol. 41, 1614-1628.

Büttner, U., Buettner, U.W., 1978. Parietal cortex (2v) neuronal activity in the alert monkey during natural vestibular and optokinetic stimulation. Brain Res. 153, 392-397.

Büttner, U., Henn, V., 1976. Thalamic unit activity in the alert monkey during natural vestibular stimulation. Brain Res. 103, 127-132.

Büttner, U., Henn, V., Oswald, H.P., 1977. Vestibular-related neuronal activity in the thalamus of the alert monkey during sinusoidal rotation in the dark. Exp. Brain Res. 30, 435-444.

Calford, M.B., 1983. The parcellation of the medial geniculate body of the cat defined by the auditory response properties of single units. J. Neurosci. 3, 2350-2364.

Carpenter, M.B., 1988. Vestibular nuclei: afferent and efferent projections. Prog. Brain Res. 76, 5-15.

Ceballos-Baumann, A.O., Boecker, H., Fogel, W., Alesch, F., Bartenstein, P., Conrad, B., Diederich, N., von Falkenhayn, I.,
Moringlane, J.R., Schwaiger, M., Tronnier, V.M., 2001. Thalamic stimulation for essential tremor activates motor and deactivates vestibular cortex. Neurology 56, 1347-1354.

Cereda, C., Ghika, J., Maeder, P., Bogousslavsky, J., 2002. Strokes restricted to the insular cortex. Neurology 59, 1950-1955.

Chen, W., Zhu, X.H., Thulborn, K.R., Ugurbil, K., 1999. Retinotopic mapping of lateral geniculate nucleus in humans using functional magnetic resonance imaging. Proc. Natl Acad. Sci. USA 96, 2430-2434.

Chen, A., DeAngelis, G.C., Angelaki, D.E., 2010. Macaque parieto-insular vestibular cortex: responses to self-motion and optic flow. J. Neurosci. 30, 3022-3042.

Colby, C.L., Duhamel, J.R., Goldberg, M.E., 1993. Ventral intraparietal area of the macaque: anatomic location and visual response properties. J. Neurophysiol. 69, 902-914.

Condé, F., Condé, H., 1978. Thalamic projections of the vestibular nuclei in the cat as revealed by retrograde transport of horseradish peroxidase. Neurosci. Lett. 9, 141-146.

Cooper, B.G., Manka, T.F., Mizumori, S.J., 2001. Finding your way in the dark: the retrosplenial cortex contributes to spatial memory and navigation without visual cues. Behav. Neurosci. 115, 1012-1028.

Craig, A.D., 2004. Distribution of trigeminothalamic and spinothalamic lamina I terminations in the macaque monkey. J. Comp. Neurol. 477, 119-148.

Craig, A.D., 2009. How do you feel-now? The anterior insula and human awareness. Nat. Rev. Neurosci. 10, 59-70.

Cullen, K.E., Roy, J.E., Sylvestre, P.A., 2003. Signal processing in vestibular nuclei: dissociating sensory, motor, and cognitive influence. In: Harris, L.R., Jenkin, M. (Eds.), Levels of perception. Springer Verlag, Berlin, pp. 285-309.

de Waele, C., Baudonnière, P.M., Lepecq, J.C., Tran Ba Huy, P., Vidal, P.P., 2001. Vestibular projections in the human cortex. Exp. Brain Res. 141, 541-551.

Deecke, L., Schwarz, D.W., Fredrickson, J.M., 1974. Nucleus ventroposterior inferior (VPI) as the ventibular thalamic relay in the rhesus monkey I. Field potential investigation. Exp. Brain Res. 20, 88-100.

Deecke, L., Schwarz, D.W., Fredrickson, J.M., 1977. Vestibular responses in the rhesus monkey ventroposterior thalamus II. Vestibulo-proprioceptive convergence at thalamic neurons. Exp. Brain Res. 30, 219-232.

Deutschländer, A., Bense, S., Stephan, T., Schwaiger, M., Brandt, T., Dieterich, M., 2002. Sensory system interactions during simultaneous vestibular and visual stimulation in PET. Hum. Brain Mapp. 16, 92-103.

Dieterich, M., Brandt, T., 1993a. Thalamic infarctions: differential effects on vestibular function in the roll plane (35 patients). Neurology 43, 1732-1740.

Dieterich, M., Brandt, T., 1993b. Ocular torsion and tilt of subjective visual vertical are sensitive brainstem signs. Ann. Neurol. 33, 292-299.

Dieterich, M., Bucher, S.F., Seelos, K.C., Brandt, T., 1998. Horizontal or vertical optokinetic stimulation activates visual motionsensitive, ocular motor and vestibular cortex areas with right hemispheric dominance. An fMRI study. Brain 121, 1479-1495.

Dieterich, M., Bense, S., Lutz, S., Drzezga, A., Stephan, T., Bartenstein, P., Brandt, T., 2003. Dominance for vestibular cortical function in the non-dominant hemisphere. Cereb. Cortex 13, 994-1007.

Dieterich, M., Bartenstein, P., Spiegel, S., Bense, S., Schwaiger, M., Brandt, T., 2005a. Thalamic infarctions cause side-specific suppression of vestibular cortex activations. Brain.

Dieterich, M., Bense, S., Stephan, T., Brandt, T., Schwaiger, M., Bartenstein, P., 2005b. Medial Vestibular Nucleus Lesions in Wallenberg's Syndrome Cause Decreased Activity of the Contralateral Vestibular Cortex. Ann. NY Acad. Sci. 1039, 368-383.

Duensing, F., Schaefer, K.P., 1958. Die Aktivität einzelner Neurone im Bereiche der Vestibulariskerne bei Horizontalbeschleunigungen 
unter besonderer Berücksichtigung des vestibulären Nystagmus. Arch Psychiatr Nervenkr Z Gesamte Neurol Psychiatr. 198, 224-252.

Duffy, C.J., 1998. MST neurons respond to optic flow and translational movement. J. Neurophysiol. 80, 1816-1827.

Duvernoy, H.M., 1999. The human brain. Surface, blood supply, and three-dimensional sectional anatomy. Second edition. Springer-Verlag, Wien.

Ebata, S., Sugiuchi, Y., Izawa, Y., Shinomiya, K., Shinoda, Y., 2004. Vestibular projection to the periarcuate cortex in the monkey. Neurosci. Res. 49, 55-68.

Eickhoff, S.B., Schleicher, A., Zilles, K., Amunts, K., 2006a. The human parietal operculum I. Cytoarchitectonic mapping of subdivisions. Cereb. Cortex 16, 254-267.

Eickhoff, S.B., Weiss, P.H., Amunts, K., Fink, G.R., Zilles, K., 2006b. Identifying human parieto-insular vestibular cortex using fMRI and cytoarchitectonic mapping. Hum. Brain Mapp. 27, 611-621.

Emri, M., Kisely, M., Lengyel, Z., Balkay, L., Marian, T., Miko, L., Berenyi, E., Sziklai, I., Tron, L., Toth, A., 2003. Cortical projection of peripheral vestibular signaling. J. Neurophysiol. 89, 2639-2646.

Engel, A.K., Moll, C.K., Fried, I., Ojemann, G.A., 2005. Invasive recordings from the human brain: clinical insights and beyond. Nat. Rev. Neurosci. 6, 35-47.

Erbayat Altay, E., Serdaroglu, A., Gucuyener, K., Bilir, E., Karabacak, N.I., Thio, L.L., 2005. Rotational vestibular epilepsy from the temporo-parieto-occipital junction. Neurology 65, 1675-1676.

Fasold, O., von Brevern, M., Kuhberg, M., Ploner, C.J., Villringer, A., Lempert, T., Wenzel, R., 2002. Human vestibular cortex as identified with caloric stimulation in functional magnetic resonance imaging. Neuroimage 17, 1384-1393.

Faugier-Grimaud, S., Ventre, J., 1989. Anatomic connections of inferior parietal cortex (area 7) with subcortical structures related to vestibulo-ocular function in a monkey (Macaca fascicularis). J. Comp. Neurol. 280, 1-14.

Fink, G.R., Marshall, J.C., Weiss, P.H., Stephan, T., Grefkes, C., Shah, N.J., Zilles, K., Dieterich, M., 2003. Performing allocentric visuospatial judgments with induced distortion of the egocentric reference frame: an fMRI study with clinical implications. Neuroimage 20, 1505-1517.

Foerster, O., 1936. Sensible corticale Felder. In: Bumke, O., Foerster, O. (Eds.), Handbuch der Neurologie. Vol. VI. Springer, Berlin, pp. 358-449.

Fredrickson, J.M., Rubin, A.M., 1986. Vestibular cortex. In: Jones, E.G., Peters, A. (Eds.), Cerebral cortex. Sensory-motor areas and aspects of cortical connectivity. Vol. 5. Plenum Press, New York, London, pp. 99-111.

Fredrickson, J.M., Figge, U., Scheid, P., Kornhuber, H.H., 1966. Vestibular nerve projection to the cerebral cortex of the rhesus monkey. Exp. Brain Res. 2, 318-327.

Friberg, L., Olsen, T.S., Roland, P.E., Paulson, O.B., Lassen, N.A., 1985. Focal increase of blood flow in the cerebral cortex of man during vestibular stimulation. Brain 108, 609-623.

Fries, W., 1984. Cortical projections to the superior colliculus in the macaque monkey: a retrograde study using horseradish peroxidase. J. Comp. Neurol. 230, 55-76.

Fukushima, K., 1997. Corticovestibular interactions: anatomy, electrophysiology, and functional considerations. Exp. Brain Res. 117, 1-16.

Fukushima, K., Kaneko, C.R., 1995. Vestibular integrators in the oculomotor system. Neurosci. Res. 22, 249-258.

Fukushima, K., Takahashi, K., Ohno, M., Kato, M., 1984. Responses of cat vestibular neurons to stimulation of the frontal cortex. Exp. Brain Res. 56, 275-278.

Fukushima, K., Sato, T., Fukushima, J., Shinmei, Y., Kaneko, C.R., 2000. Activity of smooth pursuit-related neurons in the monkey periarcuate cortex during pursuit and passive whole-body rotation. J. Neurophysiol. 83, 563-587.
Fukushima, J., Akao, T., Kurkin, S., Kaneko, C.R., Fukushima, K., 2006. The vestibular-related frontal cortex and its role in smooth-pursuit eye movements and vestibular-pursuit interactions. J. Vestib. Res. 16, 1-22.

Fukushima, K., Akao, T., Saito, H., Kurkin, S.A., Fukushima, J., Peterson, B.W., 2010. Representation of neck velocity and neck-vestibular interactions in pursuit neurons in the simian frontal eye fields. Cereb. Cortex 20, 1195-1207.

Ghazanfar, A.A., Krupa, D.J., Nicolelis, M.A., 2001. Role of cortical feedback in the receptive field structure and nonlinear response properties of somatosensory thalamic neurons. Exp. Brain Res. 141, 88-100.

Grüsser, O.J., Grüsser-Cornehls, U., 1972. Interaction of vestibular and visual inputs in the visual system. Prog. Brain Res. 37, 573-583.

Grüsser, O.J., Grüsser-Cornehls, U., Saur, G., 1959. Reaktionen einzelner Neurone im optischen Cortex der Katze nach elektrischer Polarisation des Labyrinths. Pflugers Arch. 269, 593-612.

Grüsser, O.J., Pause, M., Schreiter, U., 1982. Neural responses in the parieto-insular vestibular cortex of alert Java Monkey (Macaca fascicularis). In: Roucoux, A., Crommelinck, M. (Eds.), Physiological and pathological aspects of eye movements. Vol. Jung, The Hague, Boston, London, pp. 251-270.

Grüsser, O.J., Pause, M., Schreiter, U., 1990a. Vestibular neurones in the parieto-insular cortex of monkeys (Macaca fascicularis): visual and neck receptor responses. J. Physiol. 430, 559-583.

Grüsser, O.J., Pause, M., Schreiter, U., 1990b. Localization and responses of neurones in the parieto-insular vestibular cortex of awake monkeys (Macaca fascicularis). J. Physiol. 430, 537-557.

Grüsser, O.J., Guldin, W.O., Mirring, S., Salah-Eldin, A., 1994. Comparative physiological and anatomical studies of the primate vestibular cortex. In: Albowitz, B., Albus, K., Kuhnt, U., Nothdurf, H.C., Wahle, P. (Eds.), Structural and functional organization of the neocortex. Vol. Springer-Verlag, Berlin, pp. 358-371.

Guillery, R.W., 1995. Anatomical evidence concerning the role of the thalamus in corticocortical communication: a brief review. J. Anat. 187 (Pt 3), 583-592.

Guillery, R.W., Sherman, S.M., 2002. Thalamic relay functions and their role in corticocortical communication: generalizations from the visual system. Neuron 33, 163-175.

Guldin, W.O., Grüsser, O.J., 1998. Is there a vestibular cortex? Trends Neurosci. 21, 254-259.

Guldin, W.O., Akbarian, S., Grüsser, O.J., 1992. Cortico-cortical connections and cytoarchitectonics of the primate vestibular cortex: a study in squirrel monkeys (Saimiri sciureus). J. Comp. Neurol. 326, 375-401.

Guldin, W.O., Mirring, S., Grüsser, O.J., 1993. Connections from the neocortex to the vestibular brain stem nuclei in the common marmoset. NeuroReport 5, 113-116.

Hawrylyshyn, P.A., Rubin, A.M., Tasker, R.R., Organ, L.W., Fredrickson, J.M., 1978. Vestibulothalamic projections in man: a sixth primary sensory pathway. J. Neurophysiol. 41, 394-401.

Hegemann, S., Pawlowski, M., Huonker, R., Haueisen, J., Fitzek, C., Fetter, M., 2003. Magnetoencephalography during optokinetic and vestibular activation of the posterior insula. Ann. NY Acad. Sci. 1004, 457-464.

Hegemann, S., Fitzek, S., Fitzek, C., Fetter, M., 2004. Cortical vestibular representation in the superior temporal gyrus. J. Vestib. Res. 14, 33-35.

Hochman, M.S., 1983. Rotatory seizures associated with frontal lobe malignant neoplasm: a case report. Epilepsia 24, 11-14.

Hood, J.D., Kayan, A., 1985. Observations upon the evoked responses to natural vestibular stimulation. Electroencephalogr. Clin. Neurophysiol. 62, 266-276.

Horii, A., Russell, N.A., Smith, P.F., Darlington, C.L., Bilkey, D.K., 2004. Vestibular influences on CA1 neurons in the rat hippocampus: an electrophysiological study in vivo. Exp. Brain Res. 155, 245-250. 
Indovina, I., Maffei, V., Bosco, G., Zago, M., Macaluso, E., Lacquaniti, F., 2005. Representation of visual gravitational motion in the human vestibular cortex. Science 308, 416-419.

Isnard, J., Guenot, M., Sindou, M., Mauguiere, F., 2004. Clinical manifestations of insular lobe seizures: a stereo-electroencephalographic study. Epilepsia 45, 1079-1090.

Janzen, J., Schlindwein, P., Bense, S., Bauermann, T., Vucurevic, G., Stoeter, P., Dieterich, M., 2008. Neural correlates of hemispheric dominance and ipsilaterality within the vestibular system. Neuroimage 42, 1508-1518.

Jerbi, K., Vidal, J.R., Ossandon, T., Dalal, S.S., Jung, J., Hoffmann, D., Minotti, L., Bertrand, O., Kahane, P., Lachaux, J.P., 2010. Exploring the electrophysiological correlates of the default-mode network with intracerebral EEG. Front Syst. Neurosci. 4, 27.

Jijiwa, H., Kawaguchi, T., Watanabe, S., Miyata, H., 1991. Cortical projections of otolith organs in the cat. Acta Otolaryngol. Suppl. 481, 69-72.

Johansen-Berg, H., Behrens, T.E., Sillery, E., Ciccarelli, O., Thompson, A.J., Smith, S.M., Matthews, P.M., 2005. Functional-anatomical validation and individual variation of diffusion tractography-based segmentation of the human thalamus. Cereb. Cortex 15, 31-39.

Jones, E.G., 1985. The thalamus, Vol. Plenum Press, New York and London.

Kahane, P., Hoffmann, D., Minotti, L., Berthoz, A., 2003. Reappraisal of the human vestibular cortex by cortical electrical stimulation study. Ann. Neurol. 54, 615-624.

Karnath, H.O., Johannsen, L., Broetz, D., Kuker, W., 2005. Posterior thalamic hemorrhage induces "pusher syndrome". Neurology 64, 1014-1019.

Kempinsky, W.H., 1951. Cortical projection of vestibular and facial nerves in cat. J. Neurophysiol. 14, 203-210.

Klam, F., Graf, W., 2003. Vestibular signals of posterior parietal cortex neurons during active and passive head movements in macaque monkeys. Ann. NY Acad. Sci. 1004, 271-282.

Klam, F., Graf, W., 2006. Discrimination between active and passive head movements by macaque ventral and medial intraparietal cortex neurons. J. Physiol. 574, 367-386.

Kluge, M., Beyenburg, S., Fernandez, G., Elger, C.E., 2000. Epileptic vertigo: evidence for vestibular representation in human frontal cortex. Neurology 55, 1906-1908.

Kotchabhakdi, N., Rinvik, E., Walberg, F., Yingchareon, K., 1980. The vestibulothalamic projections in the cat studied by retrograde axonal transport of horseradish peroxidase. Exp. Brain Res. 40, 405-418.

Kurth, F., Eickhoff, S.B., Schleicher, A., Hoemke, L., Zilles, K., Amunts, K., 2010. Cytoarchitecture and probabilistic maps of the human posterior insular cortex. Cereb. Cortex 20, 1448-1461.

Lacour, M., Borel, L., 1993. Vestibular control of posture and gait. Arch. Ital. Biol. 131, 81-104.

Lai, H., Tsumori, T., Shiroyama, T., Yokota, S., Nakano, K., Yasui, Y., 2000. Morphological evidence for a vestibulo-thalamo-striatal pathway via the parafascicular nucleus in the rat. Brain Res. 872, 208-214.

Lang, W., Büttner-Ennever, J.A., Büttner, U., 1979. Vestibular projections to the monkey thalamus: an autoradiographic study. Brain Res. 177, 3-17.

Lee, C.C., Sherman, S.M., 2010. Drivers and modulators in the central auditory pathways. Front Neurosci. 4, 79.

Leinonen, L., Hyvarinen, J., Sovijarvi, A.R., 1980. Functional properties of neurons in the temporo-parietal association cortex of awake monkey. Exp. Brain Res. 39, 203-215.

Lenggenhager, B., Lopez, C., Blanke, O., 2008. Influence of galvanic vestibular stimulation on egocentric and object-based mental transformations. Exp. Brain Res. 184, 211-221.

Licata, F., Li Volsi, G., Maugeri, G., Santangelo, F., 1987. Integration of cortical and peripheral information in the lateral vestibular nucleus in the cat. Neurosci. Lett. 77, 293-297.
Licata, F., Li Volsi, G., Maugeri, G., Santangelo, F., 1990. Effects of motor cortex and single muscle stimulation on neurons of the lateral vestibular nucleus in the rat. Neuroscience 34, 379-390.

Liedgren, S.R., Schwarz, D.W., 1976. Vestibular evoked potentials in thalamus and basal ganglia of the squirrel monkey (Saimiri sciureus). Acta Otolaryngol. 81, 73-82.

Liedgren, S.R., Kristensson, K., Larsby, B., Ödkvist, L.M., 1976. Projection of thalamic neurons to cat primary vestibular cortical fields studied by means of retrograde axonal transport of horseradish peroxidase. Exp. Brain Res. 24, 237-243.

Liu, S., Dickman, J.D., Angelaki, D.E., 2010. Response Dynamics and Tilt versus Translation Discrimination in Parietoinsular Vestibular Cortex. Cereb Cortex.

Lobel, E., Kleine, J.F., Le Bihan, D., Leroy-Willig, A., Berthoz, A., 1998. Functional MRI of galvanic vestibular stimulation. J. Neurophysiol. 80, 2699-2709.

Lopez, C., Blanke, O., 2007. Neuropsychology and neurophysiology of self-consciousness. Multisensory and vestibular mechanisms. In: Holderegger, A., Sitter-Liver, B., Hess, C.W., Rager, G. (Eds.), Hirnforschung und Menschenbild. Beiträge zur interdisziplinären Verständigung. Vol. Academic Press, Schwabe, Fribourg, Basel, pp. 183-206.

Lopez, C., Lacour, M., Ahmadi, A.E., Magnan, J., Borel, L., 2007. Changes of visual vertical perception: a long-term sign of unilateral and bilateral vestibular loss. Neuropsychologia 45 , 2025-2037.

Lopez, C., Halje, P., Blanke, O., 2008. Body ownership and embodiment: Vestibular and multisensory mechanisms. Neurophysiol. Clin. 38, 149-161.

Lopez, C., Bachofner, C., Mercier, M., Blanke, O., 2009. Gravity and observer's body orientation influence the visual perception of human body postures. J. Vis. 9 (1), 1-14.

Lopez, C., Heydrich, L., Seeck, M., Blanke, O., 2010a. Abnormal self-location and vestibular vertigo in a patient with right frontal lobe epilepsy. Epilepsy Behav. 17, 289-292.

Lopez, C., Lenggenhager, B., Blanke, O., 2010b. How vestibular stimulation interacts with illusory hand ownership. Conscious. Cogn. 19, 33-47.

Maciewicz, R., Phipps, B.S., Bry, J., Highstein, S.M., 1982. The vestibulothalamic pathway: contribution of the ascending tract of Deiters. Brain Res. 252, 1-11.

Magnin, M., Fuchs, A.F., 1977. Discharge properties of neurons in the monkey thalamus tested with angular acceleration, eye movement and visual stimuli. Exp. Brain Res. 28, 293-299.

Magnin, M., Kennedy, H., 1979. Anatomical evidence of a third ascending vestibular pathway involving the ventral lateral geniculate nucleus and the intralaminar nuclei of the cat. Brain Res. 171, 523-529.

Magnin, M., Putkonen, P.T., 1978. A new vestibular thalamic area: electrophysiological study of the thalamic reticular nucleus and of the ventral lateral geniculate complex of the cat. Exp. Brain Res. 32, 91-104.

Marcelli, V., Esposito, F., Aragri, A., Furia, T., Riccardi, P., Tosetti, M., Biagi, L., Marciano, E., Di Salle, F., 2009. Spatio-temporal pattern of vestibular information processing after brief caloric stimulation. Eur. J. Radiol. 70, 312-316.

Marlinski, V., McCrea, R.A., 2008a. Activity of ventroposterior thalamus neurons during rotation and translation in the horizontal plane in the alert squirrel monkey. J. Neurophysiol. 99, 2533-2545.

Marlinski, V., McCrea, R.A., 2008b. Coding of self-motion signals in ventro-posterior thalamus neurons in the alert squirrel monkey. Exp. Brain Res. 189, 463-472.

Matelli, M., Luppino, G., 2001. Parietofrontal circuits for action and space perception in the macaque monkey. Neuroimage 14, S27-S32. 
Matesz, C., Bacskai, T., Nagy, E., Halasi, G., Kulik, A., 2002. Efferent connections of the vestibular nuclei in the rat: a neuromorphological study using PHA-L. Brain Res. Bull. 57, 313-315.

Matsuo, S., Hosogai, M., Nakao, S., 1994. Ascending projections of posterior canal-activated excitatory and inhibitory secondary vestibular neurons to the mesodiencephalon in cats. Exp. Brain Res. 100, 7-17.

Matsuo, S., Hosogai, M., Matsui, H., Ikoma, H., 1995. Posterior canal-activated vestibulocortical pathways in cats. Neurosci. Lett. 183, 131-134.

Matsuo, S., Takeuchi, H., koma, H., Hosogai, M., 1999. Location of thalamic neurons mediating vestibulo-cortical pathways in cats. Yonago Acta Med. 42, 163-166.

Matsuzaki, R., Kyuhou, S., Matsuura-Nakao, K., Gemba, H., 2004. Thalamo-cortical projections to the posterior parietal cortex in the monkey. Neurosci. Lett. 355, 113-116.

McCandless, C.H., Balaban, C.D., 2010. Parabrachial nucleus neuronal responses to off-vertical axis rotation in macaques. Exp. Brain Res. 202, 271-290.

McCormick, D.A., Bal, T., 1997. Sleep and arousal: thalamocortical mechanisms. Annu. Rev. Neurosci. 20, 185-215.

McCormick, D.A., Huguenard, J.R., 1992. A model of the electrophysiological properties of thalamocortical relay neurons. J. Neurophysiol. 68, 1384-1400.

McCrea, R.A., Gdowski, G.T., Boyle, R., Belton, T., 1999. Firing behavior of vestibular neurons during active and passive head movements: vestibulo-spinal and other non-eye-movement related neurons. J. Neurophysiol. 82, 416-428.

McGeoch, P.D., Williams, L.E., Lee, R.R., Ramachandran, V.S., 2008. Behavioural evidence for vestibular stimulation as a treatment for central post-stroke pain. J. Neurol. Neurosurg. Psychiatry 79, 1298-1301.

Melvill Jones, G., Berthoz, A., Segal, B., 1984. Adaptive modification of the vestibulo-ocular reflex by mental effort in darkness. Exp. Brain Res. 56, 149-153.

Meng, H., Bai, R.S., Sato, H., Imagawa, M., Sasaki, M., Uchino, Y., 2001. Otolith-activated vestibulothalamic neurons in cats. Exp. Brain Res. 141, 415-424.

Meng, H., May, P.J., Dickman, J.D., Angelaki, D.E., 2007. Vestibular signals in primate thalamus: properties and origins. J. Neurosci. 27, 13590-13602.

Merfeld, D.M., Zupan, L., Peterka, R.J., 1999. Humans use internal models to estimate gravity and linear acceleration. Nature 398 , 615-618.

Mergner, T., Deecke, L., Wagner, H.J., 1981. Vestibulo-thalamic projection to the anterior suprasylvian cortex of the cat. Exp. Brain Res. 44, 455-458.

Mergner, T., Becker, W., Deecke, L., 1985. Canal-neck interaction in vestibular neurons of the cat's cerebral cortex. Exp. Brain Res. 61, 94-108.

Mickle, W.A., Ades, H.W., 1954. Rostral projection pathway of the vestibular system. Am. J. Physiol. 176, 243-246.

Miller, S.M., Ngo, T.T., 2007. Studies of caloric vestibular stimulation: implications for cognitive neurosciences, the clinical neurosciences and neurophilosophy. Acta Neuropsychiatrica. 19, 183-203.

Mills, K.R., Taylor, A., 1974. The projection of the vestibular nerve to the cerebral cortex in the cat. J. Physiol. 239, 165-178.

Mittelstaedt, H., 1999. The role of the otoliths in perception of the vertical and in path integration. Ann. NY Acad. Sci. 871, 334-344.

Miyamoto, T., Fukushima, K., Takada, T., de Waele, C., Vidal, P.P., 2007. Saccular stimulation of the human cortex: a functional magnetic resonance imaging study. Neurosci. Lett. 423, 68-72.

Morel, A., Magnin, M., Jeanmonod, D., 1997. Multiarchitectonic and stereotactic atlas of the human thalamus. J. Comp. Neurol. 387, 588-630.

Morest, D.K., 1964. The Neuronal Architecture of the Medial Geniculate Body of the Cat. J. Anat. 98, 611-630.
Nagata, S., 1986. The vestibulothalamic connections in the rat: a morphological analysis using wheat germ

agglutinin-horseradish peroxidase. Brain Res. 376, 57-70.

Naito, Y., Tateya, I., Hirano, S., Inoue, M., Funabiki, K., Toyoda, H., Ueno, M., Ishizu, K., Nagahama, Y., Fukuyama, H., Ito, J., 2003. Cortical correlates of vestibulo-ocular reflex modulation: a PET study. Brain 126, 1562-1578.

Nakano, K., Kohno, M., Hasegawa, Y., Tokushige, A., 1985. Cortical and brain stem afferents to the ventral thalamic nuclei of the cat demonstrated by retrograde axonal transport of horseradish peroxidase. J. Comp. Neurol. 231, 102-120.

Nguyen, D.K., Nguyen, D.B., Malak, R., Leroux, J.M., Carmant, L., Saint-Hilaire, J.M., Giard, N., Cossette, P., Bouthillier, A., 2009. Revisiting the role of the insula in refractory partial epilepsy. Epilepsia 50, 510-520.

Nicita, F., Papetti, L., Spalice, A., Ursitti, F., Massa, R., Properzi, E., Iannetti, P., 2010. Epileptic nystagmus: Description of a pediatric case with EEG correlation and SPECT findings. J Neurol Sci.

Nishiike, S., Guldin, W.O., Bäurle, J., 2000. Corticofugal connections between the cerebral cortex and the vestibular nuclei in the rat. J. Comp. Neurol. 420, 363-372.

O'Connor, D.H., Fukui, M.M., Pinsk, M.A., Kastner, S., 2002. Attention modulates responses in the human lateral geniculate nucleus. Nat. Neurosci. 5, 1203-1209.

Ödkvist, L.M., Rubin, A.M., Schwarz, D.W., Fredrickson, J.M., 1973. Vestibular and auditory cortical projection in the guinea pig (Cavia porcellus). Exp. Brain Res. 18, 279-286.

Ödkvist, L.M., Schwarz, D.W.F., Fredrickson, J.M., Hassler, R., 1974. Projection of the vestibular nerve to the area 3a arm field in the squirrel monkey (Saimiri sciureus). Exp. Brain Res. 21, 97-105.

Ödkvist, L.M., Liedgreen, S.R., Larsby, B., Jerlvall, L., 1975. Vestibular and somatosensory inflow to the vestibular projection area in the post cruciate dimple region of the cat cerebral cortex. Exp. Brain Res. 22, 185-196.

O'Mara, S.M., Rolls, E.T., Berthoz, A., Kesner, R.P., 1994. Neurons responding to whole-body motion in the primate hippocampus. J. Neurosci. 14, 6511-6523.

Ostrowsky, K., Magnin, M., Ryvlin, P., Isnard, J., Guenot, M., Mauguiere, F., 2002. Representation of pain and somatic sensation in the human insula: a study of responses to direct electrical cortical stimulation. Cereb. Cortex 12, 376-385.

Page, W.K., Duffy, C.J., 2003. Heading representation in MST: sensory interactions and population encoding. J. Neurophysiol. 89, 1994-2013.

Papathanasiou, E.S., Papacostas, S.S., Charalambous, M., Eracleous, E., Thodi, C., Pantzaris, M., 2006. Vertigo and imbalance caused by a small lesion in the anterior insula. Electromyogr. Clin. Neurophysiol. 46, 185-192.

Pare, M., Wurtz, R.H., 1997. Monkey posterior parietal cortex neurons antidromically activated from superior colliculus. J. Neurophysiol. 78, 3493-3497.

Paus, T., 1996. Location and function of the human frontal eye-field: a selective review. Neuropsychologia 34, 475-483.

Penfield, W., 1957. Vestibular sensation and the cerebral cortex. Ann. Otol. Rhinol. Laryngol. 66, 691-698.

Penfield, W., Faulk, M.E.J., 1955. The insula: further observations on its functions. Brain 78, 445-470.

Penfield, W., Kristiansen, K., 1951. Epileptic seizure patterns, Vol. Springfield, Illinois.

Percheron, G., Francois, C., Talbi, B., Yelnik, J., Fenelon, G., 1996. The primate motor thalamus. Brain Res. Brain Res. Rev. 22, 93-181.

Petit, L., Beauchamp, M.S., 2003. Neural basis of visually guided head movements studied with fMRI. J. Neurophysiol. 89, 2516-2527.

Petit, L., Clark, V.P., Ingeholm, J., Haxby, J.V., 1997. Dissociation of saccade-related and pursuit-related activation in human frontal eye fields as revealed by fMRI. J. Neurophysiol. 77, 3386-3390. 
Potegal, M., Copack, P., de Jong, J., Krauthamer, G., Gilman, S., 1971. Vestibular input to the caudate nucleus. Exp. Neurol. 32, 448-465.

Raymond, J., Sans, A., Marty, R., 1974. Projections thalamiques des noyaux vestibulaires: étude histologique chez le chat. Exp. Brain Res. 20, 273-283.

Raymond, J., Demêmes, D., Marty, R., 1976. Voies et projections vestibulaires acsendantes émanant des noyaux vestibulaires primaires: étude radioautographique. Brain Res. 111, 1-12.

Reichova, I., Sherman, S.M., 2004. Somatosensory corticothalamic projections: distinguishing drivers from modulators. J. Neurophysiol. 92, 2185-2197.

Roucoux-Hanus, M., Boisacq-Schepens, N., 1977. Ascending vestibular projections: further results at cortical and thalamic levels in the cat. Exp. Brain Res. 29, 283-292.

Roy, J.E., Cullen, K.E., 2004. Dissociating self-generated from passively applied head motion: neural mechanisms in the vestibular nuclei. J. Neurosci. 24, 2102-2111.

Sans, A., Raymond, J., Marty, R., 1970. Réponses thalamiques et corticales à la stimulation électrique du nerf vestibulaire chez le chat. Exp. Brain Res. 10, 265-275.

Saper, C.B., 1982. Reciprocal parabrachial-cortical connections in the rat. Brain Res. 242, 33-40.

Schlack, A., Hoffmann, K.P., Bremmer, F., 2002. Interaction of linear vestibular and visual stimulation in the macaque ventral intraparietal area (VIP). Eur. J. Neurosci. 16, 1877-1886.

Schlack, A., Sterbing-D’Angelo, S.J., Hartung, K., Hoffmann, K.P., Bremmer, F., 2005. Multisensory space representations in the macaque ventral intraparietal area. J. Neurosci. 25, 4616-4625.

Schlag, J., Schlag-Rey, M., 1987. Evidence for a supplementary eye field. J. Neurophysiol. 57, 179-200.

Schlindwein, P., Mueller, M., Bauermann, T., Brandt, T., Stoeter, P., Dieterich, M., 2008. Cortical representation of saccular vestibular stimulation: VEMPs in fMRI. Neuroimage 39, 19-31.

Schlosser, H.G., Guldin, W., Fritzsche, D., Clarke, A.H., 2008. Transcranial Doppler ultrasound during galvanic labyrinth polarization depicts central vestibular processing, demonstrating bilateral vestibular projection. Eur. J. Neurosci. $28,372-378$.

Schneider, J.P., Reinohs, M., Prothmann, S., Puccini, S., Dalitz, B., Schwarz, J., Zimmer, C., Then Bergh, F., 2006. Subcortical Right Parietal AVM Rotational vertigo and caloric stimulation fMRI support a parietal representation of vestibular input. J. Neurol. 253, 253-255.

Schwarz, D.W.F., Fredrickson, J.M., 1971. Rhesus monkey vestibular cortex: a bimodal primary projection field. Science 172, 280-281.

Schwarz, D.W.F., Deecke, L., Fredrickson, J.M., 1973a. Cortical projection of group I muscle afferents to areas 2, 3a, and the vestibular field in the rhesus monkey. Exp. Brain Res. 17, 516-526.

Schwarz, D.W.F., Fredrickson, J.M., Deecke, L., 1973b. Structure and connections of the rhesus vestibular cortex. Adv. Otorhinolaryngol. 19, 206-209.

Selimbeyoglu, A., Parvizi, J., 2010. Electrical stimulation of the human brain: perceptual and behavioral phenomena reported in the old and new literature. Front Hum Neurosci. 4, 46.

Sherman, S.M., 2001. Tonic and burst firing: dual modes of thalamocortical relay. Trends Neurosci. 24, 122-126.

Sherman, S.M., 2005. Thalamic relays and cortical functioning. Prog. Brain Res. 149, 107-126.

Sherman, S.M., Guillery, R.W., 1998. On the actions that one nerve cell can have on another: distinguishing "drivers" from "modulators". Proc. Natl Acad. Sci. USA 95, 7121-7126.

Sherman, S.M., Guillery, R.W., 2002. The role of the thalamus in the flow of information to the cortex. Philos. Trans. R. Soc. Lond. B Biol. Sci. 357, 1695-1708.

Shiroyama, T., Kayahara, T., Yasui, Y., Nomura, J., Nakano, K., 1995. The vestibular nuclei of the rat project to the lateral part of the thalamic parafascicular nucleus (centromedian nucleus in primates). Brain Res. 704, 130-134.

Shiroyama, T., Kayahara, T., Yasui, Y., Nomura, J., Nakano, K., 1999. Projections of the vestibular nuclei to the thalamus in the rat: a Phaseolus vulgaris leucoagglutinin study. J. Comp. Neurol. 407, 318-332.

Sillito, A.M., Cudeiro, J., Jones, H.E., 2006. Always returning: feedback and sensory processing in visual cortex and thalamus. Trends Neurosci. 29, 307-316.

Smith, B.H., 1960. Vestibular disturbances in epilepsy. Neurology $10,465-469$.

Smith, P.F., 1997. Vestibular-hippocampal interactions. Hippocampus 7, 465-471.

Smith, P.F., Zheng, Y., Horii, A., Darlington, C.L., 2005. Does vestibular damage cause cognitive dysfunction in humans? J. Vestib. Res. 15, 1-9.

Smith, P.F., Darlington, C.L., Zheng, Y., 2010. Move it or lose it-is stimulation of the vestibular system necessary for normal spatial memory? Hippocampus 20, 36-43.

Spiegel, E.A., Szekely, E.G., Gildenberg, P.L., 1965. Vestibular Responses in Midbrain, Thalamus, and Basal Ganglia. Arch. Neurol. 12, 258-269.

Squire, L.R., Bloom, F.E., McDonnell, S.K., Roberts, J.L., Spitzer, N.C., Zigmond, M.J., 2008. Fundamental neuroscience, Vol. Academic Press, Amsterdam.

Stephan, T., Deutschländer, A., Nolte, A., Schneider, E., Wiesmann, M., Brandt, T., Dieterich, M., 2005. Functional MRI of galvanic vestibular stimulation with alternating currents at different frequencies. Neuroimage 26, 721-732.

Straube, A., Brandt, T., 1987. Importance of the visual and vestibular cortex for self-motion perception in man (circularvection). Hum. Neurobiol. 6, 211-218.

Sugiuchi, Y., Izawa, Y., Ebata, S., Shinoda, Y., 2005. Vestibular cortical area in the periarcuate cortex: its afferent and efferent projections. Ann. NY Acad. Sci. 1039, 111-123.

Suzuki, M., Kitano, H., Ito, R., Kitanishi, T., Yazawa, Y., Ogawa, T., Shiino, A., Kitajima, K., 2001. Cortical and subcortical vestibular response to caloric stimulation detected by functional magnetic resonance imaging. Brain Res. Cogn. Brain Res. 12, 441-449.

Taube, J.S., 1998. Head direction cells and the neurophysiological basis for a sense of direction. Prog. Neurobiol. 55, 225-256.

Tehovnik, E.J., Sommer, M.A., Chou, I.H., Slocum, W.M., Schiller, P.H., 2000. Eye fields in the frontal lobes of primates. Brain Res. Brain Res. Rev. 32, 413-448.

Temereanca, S., Simons, D.J., 2004. Functional topography of corticothalamic feedback enhances thalamic spatial response tuning in the somatosensory whisker/barrel system. Neuron 41, 639-651.

Thier, P., Erickson, R.G., 1992. Responses of Visual-Tracking Neurons from Cortical Area MST-I to Visual, Eye and Head Motion. Eur. J. Neurosci. 4, 539-553.

Tiecks, F.P., Planck, J., Haberl, R.L., Brandt, T., 1996. Reduction in posterior cerebral artery blood flow velocity during caloric vestibular stimulation. J. Cereb. Blood Flow Metab. 16, 1379-1382.

Tomlinson, R.D., Robinson, D.A., 1984. Signals in vestibular nucleus mediating vertical eye movements in the monkey. J. Neurophysiol. 51, 1121-1136.

Trousselard, M., Barraud, P.A., Nougier, V., Raphel, C., Cian, C., 2004. Contribution of tactile and interoceptive cues to the perception of the direction of gravity. Brain Res. Cogn. Brain Res. 20, 355-362.

Tuohimaa, P., Aantaa, E., Toukoniitty, K., Makela, P., 1983. Studies of vestibular cortical areas with short-living $15 \mathrm{O} 2$ isotopes. ORL J. Otorhinolaryngol. Relat. Spec. 45, 315-321.

Urasaki, E., Yokota, A., 2006. Rotational vertigo caused by cerebral lesions: vertigo and areas $3 \mathrm{av}, 2 \mathrm{v}$, and 7. J. Clin. Neurosci. 13, 114-116. 
Vallar, G., Bottini, G., Rusconi, M.L., Sterzi, R., 1993. Exploring somatosensory hemineglect by vestibular stimulation. Brain 116 (Pt 1), 71-86.

Vanni-Mercier, G., Magnin, M., 1982. Single neuron activity related to natural vestibular stimulation in the cat's visual cortex. Exp. Brain Res. 45, 451-455.

Ventre, J., Faugier-Grimaud, S., 1986. Effects of posterior parietal lesions (area 7) on VOR in monkeys. Exp. Brain Res. 62, 654-658.

Ventre, J., Faugier-Grimaud, S., 1988. Projections of the temporo-parietal cortex on vestibular complex in the macaque monkey (Macaca fascicularis). Exp. Brain Res. 72, 653-658.

Ventre-Dominey, J., Nighoghossian, N., Denise, P., 2003. Evidence for interacting cortical control of vestibular function and spatial representation in man. Neuropsychologia 41, 1884-1898.

Vitte, E., Derosier, C., Caritu, Y., Berthoz, A., Hasboun, D., Soulie, D., 1996. Activation of the hippocampal formation by vestibular stimulation: a functional magnetic resonance imaging study. Exp. Brain Res. 112, 523-526.

Walzl, E.M., Mountcastle, V.B., 1949. Projection of vestibular nerve to cerebral cortex of the cat. Am. J. Physiol. 159, 595.

Wenzel, R., Bartenstein, P., Dieterich, M., Danek, A., Weindl, A., Minoshima, S., Ziegler, S., Schwaiger, M., Brandt, T., 1996. Deactivation of human visual cortex during involuntary ocular oscillations. A PET activation study. Brain. 119 (Pt 1), 101-110.
Wiener, S.I., Berthoz, A., Zugaro, M.B., 2002. Multisensory processing in the elaboration of place and head direction responses by limbic system neurons. Brain Res. Cogn. Brain Res. 14, 75-90.

Wiest, G., Zimprich, F., Prayer, D., Czech, T., Serles, W., Baumgartner, C., 2004. Vestibular processing in human paramedian precuneus as shown by electrical cortical stimulation. Neurology 62 , 473-475.

Wilson, V.J., Melvill Jones, G., 1979. Mammalian vestibular physiology, Vol. Plenum Press, New York, London.

Wilson, V.J., Zarzecki, P., Schor, R.H., Isu, N., Rose, P.K., Sato, H., Thomson, D.B., Umezaki, T., 1999. Cortical influences on the vestibular nuclei of the cat. Exp. Brain Res. 125, 1-13.

Woollacott, M., Shumway-Cook, A., 2002. Attention and the control of posture and gait: a review of an emerging area of research. Gait Posture 16, 1-14.

Zhang, D., Snyder, A.Z., Fox, M.D., Sansbury, M.W., Shimony, J.S., Raichle, M.E., 2008. Intrinsic functional relations between human cerebral cortex and thalamus. J. Neurophysiol. 100, 1740-1748.

Zwergal, A., Buttner-Ennever, J., Brandt, T., Strupp, M., 2008. An ipsilateral vestibulothalamic tract adjacent to the medial lemniscus in humans. Brain 131, 2928-2935.

Zwergal, A., Strupp, M., Brandt, T., Buttner-Ennever, J.A., 2009. Parallel ascending vestibular pathways: anatomical localization and functional specialization. Ann. NY Acad. Sci. 1164, 51-59. 